\title{
2. DIAGENESIS OF CLAY AND SILICA MINERALS IN SEDIMENTS FROM THE ARGO BASIN, NORTHEASTERN INDIAN OCEAN (SITE 765) ${ }^{1}$
}

\author{
John S. Compton ${ }^{2}$ and Stanley D. Locker ${ }^{2}$
}

\begin{abstract}
Sediments recovered from Site 765 can be divided into seven mineral associations, based on differences in clay mineralogy. These clay mineral associations correlate with the lithologic units and reflect the rift-to-drift history of the passive Australian margin. In general, the Lower to mid-Cretaceous sediments represent altered volcanic material and detrital aluminosilicates that were deposited during the early formation of the Argo Basin. The predominant clay mineral is randomly interstratified illite/smectite (I/S) that contains less than $10 \%$ illite layers. The transformation of smectite to illite is suggested by an increase in the percentage of illite layers in the basal sediments (from $<10 \%$ to $40 \%$ ) that corresponds to the silica transformation of opal-CT to quartz. This mixed-layered illite/smectite has an average composition of $\left(\mathrm{K}_{0.14} \mathrm{Na}_{0.29} \mathrm{Ca}_{0.07}\right)\left(\mathrm{Al}_{0.88} \mathrm{Mg}_{0.43} \mathrm{Fe}_{0.61} \mathrm{Ti}_{0.06}\right)\left(\mathrm{Si}_{3.88} \mathrm{Al}_{0.12}\right)(\mathrm{O})_{10}(\mathrm{OH})_{2}$. The highly smectitic composition of the $\mathrm{I} / \mathrm{S}$ and its association with bentonite layers and zeolite minerals suggest that much of the $\mathrm{I} / \mathrm{S}$ was derived from the alteration of volcanic material.

The condensed middle to Upper Cretaceous sediments consist of palygorskite and detrital I/S that contains $30 \%$ to $60 \%$ illite layers. The condensed Paleogene sediments contain no palygorskite and are dominated by detrital clay minerals or by highly smectitic I/S associated with bentonite layers and zeolite minerals. The overlying, rapidly deposited Neogene clayey calcareous turbidites consist of three distinct clay mineral associations. Middle Miocene sediments contain palygorskite, kaolinite, and a tentatively identified mixed-layered illite/smectite/chlorite (I/S/C) or saponite. Upper Miocene sediments contain abundant sepiolite and kaolinite and lesser amounts of detrital I/S. Detrital I/S and kaolinite dominate the clay mineralogy of Pliocene and Pleistocene sediments. The fibrous, magnesium-rich clay minerals sepiolite and palygorskite appear to be authigenic and occur intimately associated with authigenic dolomite. The magnesium required to form these $\mathrm{Mg}$-rich minerals was supplied by diffusion from the overlying seawater, and silica was supplied by the dissolution of associated biogenic silica.
\end{abstract}

\section{INTRODUCTION}

One of the key objectives of Leg 123 was to document the rift-to-drift evolution of the Australian continental margin. Changes in the mineral composition of the sediments, particularly the authigenic and detrital clay minerals, can provide important information for interpreting original sediment composition, burial diagenesis and changes in the sediment source area. Site 765 is located on oceanic basement directly on the edge of the passive trailing margin of the Australian continent (Fig. 1). The age of the oceanic basement at Site 765 is not known with certainty, but the basal sediments are dated as Tithonian (Kaminski et al., this volume). The Argo Basin currently is a deep abyssal basin, where Site 765 is located at a water depth of $5740 \mathrm{~m}$. The objectives of this study are (1) to document the mineral composition of the sediments recovered at Site $765,(2)$ to determine the burial diagenesis of the sediments at Site 765 by relating changes in sediment mineralogy to trends in pore-water chemistry, and (3) to relate changes in mineralogy to changes in the lithology and age of the sediments to understand the tectonic evolution of the Argo Basin better.

\section{METHODS}

Samples were collected on board the JOIDES Resolution during Leg 123. Bulk samples were scraped clean of any contamination from drilling mud, placed in beakers with deionized water, and disaggregated with a rubber spatula. Dispersant (approximately $20 \mathrm{~mL}$ of $5 \mathrm{wt} \%$ sodium hexametaphosphate solution) was

\footnotetext{
${ }^{1}$ Gradstein, F. M., Ludden, J. N., et al., 1992. Proc. ODP, Sci. Results, 123: College Station, TX (Ocean Drilling Program).

Department of Marine Science, University of South Florida, St. Petersburg, FL 33701, U.S.A.
}

added to each sample to prevent flocculation of the clay minerals. The dispersed sample was ultrasonicated in a bath for several minutes until the sediment particles had disaggregated and were suspended in a slurry. This slurry was washed through a $38-\mu \mathrm{m}$ stainless-steel sieve and the collected $>38-\mu \mathrm{m}$ size fraction was dried overnight at $60^{\circ} \mathrm{C}$. The $<38-\mu \mathrm{m}$ slurry was centrifuged to settle particles $>2-\mu \mathrm{m}$ in spherical diameter. The $<2-\mu \mathrm{m}$ slurry was centrifuged to settle particles $>0.1 \mu \mathrm{m}$ in diameter. The 0.1 to $2-\mu \mathrm{m}$ size fraction typically consisted of a dark, greasy top layer (approximately $<0.5 \mu \mathrm{m}$ ) and a light, sticky base layer. A glass smear slide was made for the $>38-, 2-$ to $38-,<2-\mu$ m base layer, and the $<2-\mu \mathrm{m}$ top layer and run on a Scintag XDS $2000 \mathrm{X}$-ray diffractometer (XRD) using $\mathrm{Cu} K \alpha$ radiation. The $<2-\mu \mathrm{m}$ size fraction was soaked in ethylene glycol overnight and rerun on the XRD. The percentage of interstratified illite in the mixed-layered illite/smectite (I/S) was determined by measuring the position of the $002 / 003$ reflection and by using the techniques described by Reynolds and Hower (1970), Reynolds (1980), and Moore and Reynolds (1989). The $>38-\mu \mathrm{m}$ size fraction was examined using a binocular microscope, and selected bulk samples were examined using an ISI D-130 scanning electron microscope (SEM). The major- and trace-element chemistry of the bulk samples was determined on board the ship by XRF analysis and by T. Plank at Lamont-Doherty Geological Observatory, Columbia University (Plank and Ludden, this volume).

\section{MINERALOGY OF SITE 765}

The mineralogy of Site 765 can be divided into seven distinct mineral associations based primarily on differences in their clay mineralogy. The relative abundance of the minerals observed at Site 765 is presented in Table 1 . The seven mineral associations are described below in the order in which they were deposited, starting with the oldest sediments (mineral Association VII) and finishing with the youngest sediments (mineral Association I) 


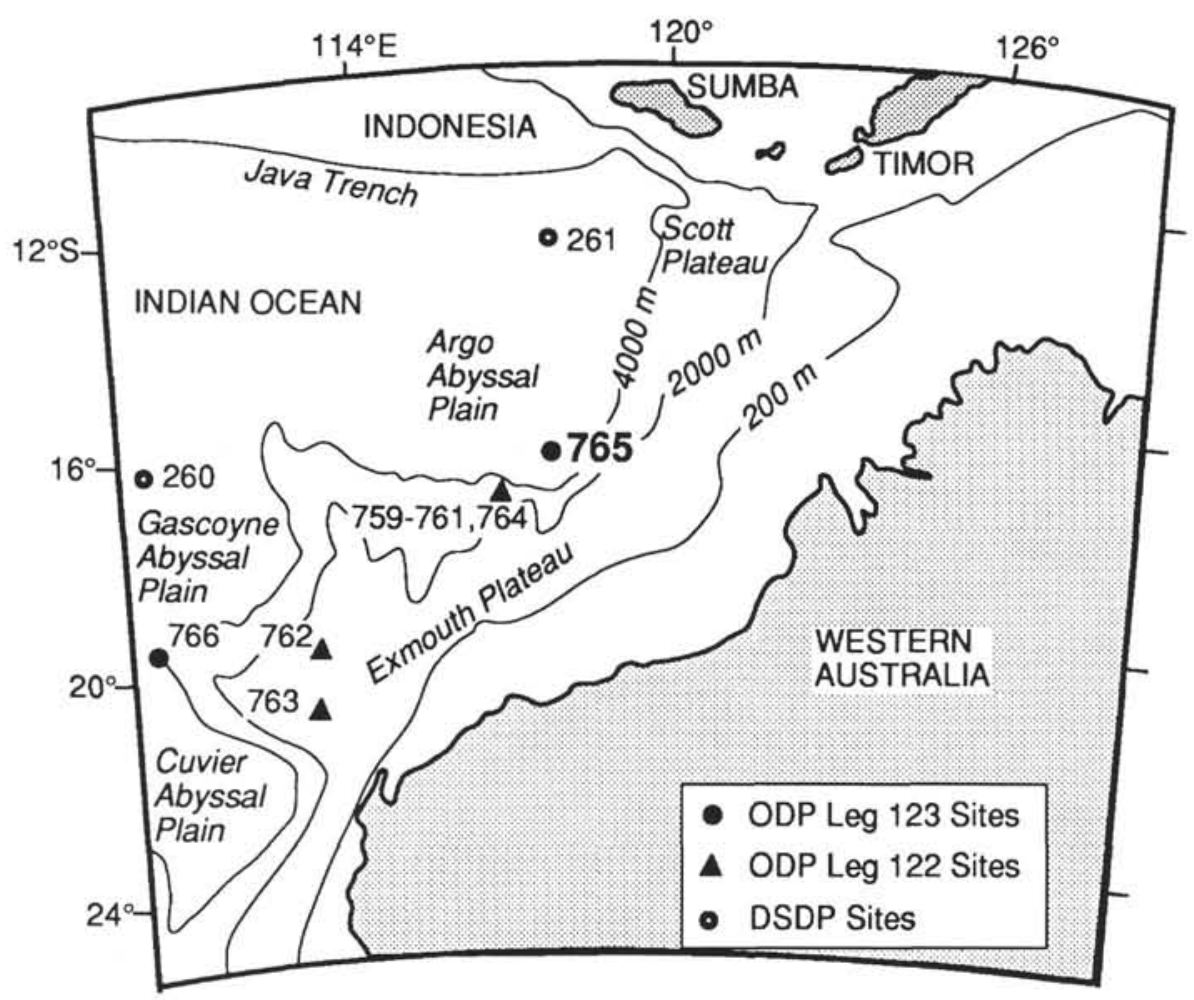

Figure 1. Location of Site 765 in the Argo Abyssal Plain, northeastern Indian Ocean.

recovered at Site 765 . The correlation among mineral associations, location of analyzed samples, sediment depth, age, and lithology are shown in Figure 2.

\section{Mineral Association VII}

Mineral Association VII includes sediment from 930 to $912 \mathrm{~m}$ below seafloor (mbsf), ranges in age from Tithonian to Valanginian, and corresponds to lithologic Unit VII. Lithologic Unit VII consists of dark reddish-brown, noncalcareous claystone and brown silty claystone with calcareous shell fragments, radiolarites, and several bentonite layers (von Rad and Thurow, in press). For example, Sample 123-765C-60R-5, $120 \mathrm{~cm}$, was interpreted as a bentonite layer (Ludden, Gradstein, et al., 1990). Mineral Association VII consists of randomly interstratified illite/smectite (I/S) having approximately $17 \%$ to $40 \%$ illite layers (Fig. 3A). The smectite mineral species is montmorillonite, based on the position of the 060 reflection (e.g., Moore and Reynolds, 1989). The commonly observed texture of sediment from mineral Association VII is shown in the SEM photomicrographs of Sample 123-765C-61R-5, $81 \mathrm{~cm}$, containing I/S with about $40 \%$ illite layers (Pl. 1, Figs. 1 and 2). The I/S is in contact with the rounded surface of a detrital grain, and the close-up shows the wavy flakes typical of $\mathrm{I} / \mathrm{S}$, where the flake edges are scalloped into fairly sharp points (Keller et al., 1986). Along with $\mathrm{I} / \mathrm{S}$, these samples contain varying amounts of detrital quartz, quartz-replaced radiolarians (radiolarites), feldspar minerals (predominantly K-feldspar), discrete illite, and mica (Table 1). Minor-to-trace amounts of pyrite and trace amounts of dolomite were observed in this interval. Mineral Association VII is the only mineral association at Site 765 that contains no detectable kaolinite by XRD analysis.

\section{Mineral Association VI}

Lower to mid-Cretaceous sediments recovered from 896 to 638 mbsf are composed predominantly of $\mathrm{I} / \mathrm{S}$ that contains $10 \%$ or less interstratified illite layers (Figs. $3 \mathrm{~B}$ and $3 \mathrm{C}$ ). The much more smectitic composition of the I/S distinguishes mineral Association VI from the underlying mineral Association VII. The I/S has a wavy flake texture commonly observed for highly smectitic I/S (Sample 123-765C-51R-3, 140-150 cm; PI. 1, Figs. 3 and 4). In addition to $\mathrm{I} / \mathrm{S}$, these sediments contain varying amounts of detrital quartz, K-feldspar and plagioclase feldspar, illite, and mica (Table 1). Abundant, quartz-replaced radiolarians occur below $740 \mathrm{mbsf}$, and opal-CT occurs from about 660 to $740 \mathrm{mbsf}$. The opal-CT occurs as lepispheres intimately associated with highly smectitic I/S (Pl. 2, Figs. 1 and 2). Minor-to-trace amounts of kaolinite, pyrite, and dolomite occur in places in mineral Association VI.

Mineral Association VI corresponds to lithologic Units VI, V, and IV (Subunits B, C, and D). Lithologic Unit VI is predominantly calcareous chalk with interbedded claystones, radiolarites, bentonites, and rhodochrosite-bearing sediment. Rhodochrosite occurs in Sample 123-765-45R-3, 140-150 cm, and calcispheres were observed in Sample 123-765-57R-3, 140-150 cm. Lithologic Unit V contains far less carbonate than Unit VI and consists mostly of claystones, as well as some radiolarites and rhodochrosite-bearing sediments. The transition from mineral Association VI to Association V corresponds to a change in color within lithologic Subunit IVB at 640 mbsf. Sediments below $640 \mathrm{mbsf}$ are mostly gray and green and contain more carbonate than the mostly red and brown sediments above $640 \mathrm{mbsf}$.

\section{Mineral Association V}

Mineral Association V (631-570 mbsf) consists of random I/S that contains variable amounts of interstratified illite layers $(28 \%-58 \%)$, palygorskite, kaolinite, and lesser amounts of illite and mica. The kaolinite, illite, and mica are concentrated in the $>2-\mu \mathrm{m}$ size fraction, along with nannofossils, foraminifers, quartz, and feldspar. Trace amounts of dolomite occur in this interval, and pyrite is rare. Clinoptilolite is common in minor amounts, especially in lithologic Subunit IVA. The red and brown 
sediments of mineral Association $\mathrm{V}$ range in age from middle to Late Cretaceous and consist of claystones (zeolitic in places), clayey nannofossil chalks, and calcareous turbidites (lithologic Subunits IVB, IVA, and IIIC) (Ludden, Gradstein, et al., 1990).

\section{Mineral Association IV}

Mineral Association IV ranges in age from Paleocene through early Miocene (553-447 mbsf) and can be subdivided into two parts (dashed line in Fig. 2). The lower part (Cores 123-765C-22R through $-18 \mathrm{R}$, Paleocene and Eocene) is composed predominantly of highly smectitic I/S ( $<10 \%$ illite layers) and kaolinite, with minor-to-trace amounts of illite, mica, quartz, and feldspar. Clinoptilolite is common in the lower part. An XRD pattern of the $<0.5-\mu \mathrm{m}$ size fraction from a sample between mineral Association $\mathrm{V}$ and the lower part of mineral Association IV is shown in Figure 4. The $\mathrm{I} / \mathrm{S}$ of the upper part (Cores 123-765C-18R through -13R, Oligocene and lower Miocene) is less smectitic and contains $12 \%$ to $40 \%$ illite layers. Trace amounts of dolomite occur in places, but no palygorskite was found in mineral Association IV. Mineral Association IV corresponds to lithologic Subunits IIIB, IIIA, and IIC, which consist of interbedded, varicolored claystones and graded clayey nannofossil oozes. Nannofossils, foraminifers, quartz, feldspar, mica, and organic matter occur in the $>2-\mu \mathrm{m}$ size fraction.

\section{Mineral Association III}

Mineral Association III (441-368 mbsf) comprises the middle Miocene and is dominated by palygorskite, kaolinite, and an unusual blue-green clay mineral in the $<0.5-\mu \mathrm{m}$ size fraction that was tentatively identified as randomly interstratified illite/smectite/chlorite (I/S/C) or saponite, based on a comparison of the glycolated XRD patterns (Fig. 5) with calculated XRD patterns presented in Reynolds (1980) (see below). SEM photomicrographs of a sample from mineral Association III are shown in Plate 2 , Figures 3 and 4 . The presence of kaolinite was confirmed by the disappearance of the 7.21 and $3.58 \AA$ reflections after heating the samples to $550^{\circ} \mathrm{C}$ for $1 \mathrm{hr}$ (Fig. 5). Discrete illite and mica are concentrated in the $>2-\mu \mathrm{m}$ size fraction. The $2-$ to $38-\mu \mathrm{m}$ size fraction contains abundant calcian dolomite that occurs as disseminated euhedral rhombic crystals (Compton, this volume). Fibrous palygorskite is commonly observed attached to dolomite crystal surfaces (Pl. 3, Figs. 1 and 2). Mineral Association III corresponds to lithologic Subunit IIB, which consists of graded sequences of clayey nannofossil ooze interbedded with green claystones. Nannofossils, calcitic and aragonitic foraminifers, quartz, dolomite, and pyrite occur in the $>2-\mu \mathrm{m}$ size fraction. These samples appear to contain few, if any, feldspar minerals.

Mineral Association II was differentiated from mineral Association III by the presence of sepiolite and the absence of saponite-I/S/C. However, the transition from mineral Associations III to II is gradational. For example, both sepiolite and saponite-I/S/C were found in Sample 123-765B-39X-1, $86 \mathrm{~cm}$, at the boundary between mineral Associations III and II ( $368 \mathrm{mbsf}$ ), and abundant saponite-I/S/C was found to occur in at least one sample from mineral Association II (Sample 123-765B-33X-5, 9 cm, Fig. 5B).

\section{Mineral Association II}

Mineral Association II (368-196 mbsf) comprises the upper Miocene sediments and consists of sepiolite, kaolinite, I/S, palygorskite, mite, and mica (Table 1). Sepiolite is the most abundant clay mineral in the $<0.5-\mu \mathrm{m}$ size fraction (Fig. 6). Kaolinite is concentrated in the 0.5 - to $2-\mu \mathrm{m}$ size fraction, along with lesser amounts of illite and mica. Samples contain approximately equal amounts of I/S and palygorskite, both of which are less abundant than kaolinite. The I/S contains variable amounts of randomly interstratified illite (30\% to $65 \%$ ). Mineral Association II is dominated by graded calcareous sequences (lithologic Subunit IIA), but has fewer intercalated clay-rich beds than mineral Association III. The $>2-\mu \mathrm{m}$ size fraction is composed mostly of nannofossils, foraminifers, quartz, pyrite, and organic matter (Sample 123$765 \mathrm{~B}-36 \mathrm{X}-2,140-150 \mathrm{~cm}$, contains coal). Similar to mineral Association III, these sediments appear to contain only a trace amount of feldspar minerals. These sediments are highly calcareous and contain an average of $70 \mathrm{wt} \% \mathrm{CaCO}_{3}$. Aragonitic foraminifers and disseminated dolomite rhombs are common.

\section{Mineral Association I}

Mineral Association I is early Pliocene to Holocene in age and comprises the uppermost sediments at Site 765 (177-0 mbsf). It contains approximately equal amounts of I/S and kaolinite, with lesser amounts of illite and mica. The I/S is concentrated in the $<0.5-\mu \mathrm{m}$ size fraction and has variable amounts of randomly interstratified illite. The amount of interstratified illite is difficult to determine because the XRD patterns and, in particular, the $002 / 003$ reflection are poorly defined because of a broad peak centered around $4.1 \AA$ from the presence of X-ray amorphous opal-A. However, the overall XRD pattern suggests that the I/S contains $>20 \%$ illite layers. Mineral Association I consists of pelagic calcareous turbidites with minor intercalated clayey sediment (lithologic Unit I). The relative abundance of clay minerals varies depending on the sample position within the graded calcareous sequences, but the clay mineralogy is similar throughout the sediment of lithologic Unit I. The sediments are less calcareous than underlying mineral Association II and contain an average of $47 \mathrm{wt} \% \mathrm{CaCO}_{3}$ in the form of nannofossils and foraminifers (Ludden, Gradstein, et al., 1990). Aragonitic foraminifers are present, but are far less abundant than in mineral Associations II and III and tend to be concentrated in the basal sand layers of the turbidites. Detrital quartz, K-feldspar and plagioclase feldspar, mica, and organic matter were observed in the $>2-\mu \mathrm{m}$ size fractions. Pyrite framboids (silt-sized spheres of aggregated pyrite crystals) and pyritized burrows also were observed in the $>2-\mu \mathrm{m}$ size fraction. A single calcareous nodule in Sample 123-765B$6 \mathrm{H}-3,145-150 \mathrm{~cm}$, contains the mineral francolite, a carbonate fluorapatite. Opal-A is present as abundant, well-preserved radiolarian tests from 90 to 0 mbsf. Trace-to-minor amounts of dolomite were found below $62 \mathrm{mbsf}$.

\section{Structural Formulas of the Clay Minerals}

The elemental composition of clay mineral separates $(<2-\mu \mathrm{m}$ size fraction) from five samples was determined on board the Resolution by X-ray fluorescence spectroscopy (XRF). The approximate mineral composition of the samples was determined by combining the semiquantitative mineral abundances from the XRD patterns (based on relative peak areas) and the major oxide elemental analyses (Table 2). The clay mineral separates analyzed are predominantly mixed-layered I/S, with varying amounts of quartz, kaolinite, illite, and calcite. Calculation of the structural formulas (Table 3) assumed anion-cation balance and an ideal structure containing $\mathrm{O}_{10}(\mathrm{OH})_{2}$ (Newman and Brown, 1987). Total $\mathrm{Fe}\left(\mathrm{FeO}\right.$ and $\mathrm{Fe}_{2} \mathrm{O}_{3}$ ) was measured as $\mathrm{Fe}_{2} \mathrm{O}_{3}$. Fe oxide coatings on the clay minerals were assumed to be negligible. Excess $\mathrm{Al}$ was accounted for by kaolinite, if present, and excess silica was accounted for by quartz. The calculated structural formulas are only approximate because the abundance of illite, kaolinite, and quartz and the elemental composition of the illite are approximate.

The structural formulas provide insight about the composition of the I/S clay minerals from the Cretaceous sediments at Site 765. The calculated clay formulas are consistent with the XRD results that these I/S clay minerals are highly smectitic and contain less 
Table 1. Mineralogy of sediment recovered at Site 765 .

\begin{tabular}{|c|c|c|c|c|c|c|c|c|c|c|c|c|c|c|c|c|c|c|c|c|c|c|c|}
\hline \multirow[b]{2}{*}{ M.A. } & \multirow[b]{2}{*}{ Core $(\mathrm{cm})$} & \multirow[b]{2}{*}{$\begin{array}{l}\text { Depth } \\
\text { (mbsf) }\end{array}$} & \multirow[b]{2}{*}{ Color } & \multirow[b]{2}{*}{ Lithology } & \multicolumn{3}{|c|}{ Silica } & \multicolumn{3}{|c|}{ Carbonate } & \multirow{2}{*}{$\begin{array}{c}\text { Org. } \\
\text { Ca } \\
\text { (wt\%) }\end{array}$} & \multicolumn{2}{|c|}{ Feldspar } & \multicolumn{10}{|c|}{ Clay minerals $(<2 \mu \mathrm{m})$} \\
\hline & & & & & Qtz & $0-A$ & O-CT & $\begin{array}{l}\mathrm{CaCO}_{3} \\
(\%)\end{array}$ & Arag. & Dol. & & K & Plag & Mica & Illite & Kaol & Sep & Palyg & $\mathrm{I} / \mathrm{S} / \mathrm{C}$ & $\mathrm{I} / \mathrm{S}$ & $\% \mathrm{I}$ & Pyr & Zeol \\
\hline 1 & $2 \mathrm{H}-3,145$ & 13.8 & Green & Radiolarian clay & 5 & 6 & 0 & 0.8 & 0 & 0 & 1.08 & 1 & 1 & 3 & 4 & 5 & 0 & 0 & 0 & 6 & $>20$ & 0 & 0 \\
\hline i & $2 \mathrm{H}-6.140$ & 18.2 & Dark gray/green & Radiolarian clay & 6 & 6 & 0 & 0.6 & 0 & 0 & 0.72 & 2 & 2 & 0 & 4 & 4 & 0 & 0 & 0 & 6 & $>20$ & 0 & 0 \\
\hline i & $4 \mathrm{H}-3,145$ & 33.0 & White/green & $\begin{array}{l}\text { Foraminifera/ } \\
\text { radiolarian ooze }\end{array}$ & 4 & 5 & 0 & 56.6 & 2 & 0 & 0.94 & 0 & 0 & i & 4 & 5 & 0 & 0 & 0 & 6 & $>20$ & 0 & 0 \\
\hline I & $4 \mathrm{H}-6,23$ & 36.2 & Dark red-gray & Clay & 6 & 5 & 0 & 2.4 & 0 & 0 & 0.91 & 2 & 2 & 0 & 4 & 4 & 0 & 0 & 0 & 5 & $>20$ & 0 & 0 \\
\hline 1 & $6 \mathrm{H}-3,145$ & 52.3 & Gray/green & Nannofossil ooze & 4 & 4 & 0 & 62.1 & 0 & 0 & 0.51 & 0 & 0 & 2 & 3 & 5 & 0 & 0 & 0 & 6 & $>20$ & 2 & 0 \\
\hline i & $7 \mathrm{H}-3,38$ & 60.8 & $5 Y 6 / 1$ & $\begin{array}{l}\text { Clayey nannofossil } \\
\text { ooze }\end{array}$ & 5 & 0 & 0 & 70.3 & 1 & 1 & 0.58 & 2 & 2 & 1 & 4 & 3 & 0 & 0 & 0 & 4 & $>20$ & 0 & 0 \\
\hline I & $8 \mathrm{H}-3,145$ & 71.6 & Green & $\begin{array}{l}\text { Clayey nannofossil } \\
\text { ooze }\end{array}$ & 3 & 4 & 0 & 70.9 & 2 & 1 & 0.97 & 0 & 0 & 1 & 3 & 6 & 0 & 0 & 0 & 4 & $>20$ & 0 & 0 \\
\hline 1 & $8 \mathrm{H}-6,85$ & 75.5 & $5 \mathrm{Y} 6 / 2$ & Calcareous clay & 4 & 0 & 0 & 46.9 & 2 & 2 & 0.29 & 1 & 1 & 0 & 3 & 3 & 0 & 0 & 0 & 5 & $>20$ & 2 & 0 \\
\hline I & $10 \mathrm{H}-3,145$ & 90.9 & Pale green & $\begin{array}{l}\text { Clayey nannofossil } \\
\text { ooze }\end{array}$ & 4 & 4 & 0 & 57.9 & 0 & 1 & 0.27 & 0 & 0 & 0 & 3 & 4 & 0 & 0 & 0 & 5 & $>20$ & 2 & 0 \\
\hline I & $12 \mathrm{H}-3,145$ & 110.1 & Pale green & $\begin{array}{l}\text { Clayey nannofossil } \\
\text { ooze }\end{array}$ & 4 & 0 & 0 & 72.2 & 0 & 1 & 0.08 & 0 & 0 & 2 & 3 & 6 & 0 & 0 & 0 & 5 & $>20$ & 0 & 0 \\
\hline I & $13 \mathrm{H}-2,138$ & 118.1 & $5 Y 6 / 2$ & $\begin{array}{l}\text { Clayey nannofossil } \\
\text { ooze }\end{array}$ & 4 & 0 & 0 & 60.9 & 2 & 1 & 0.31 & 0 & 0 & 0 & 5 & 4 & 0 & 0 & 0 & 6 & $>20$ & 0 & 0 \\
\hline I & $15 \mathrm{H}-2,88$ & 136.9 & $5 Y 4 / 1$ & Clay & 4 & 0 & 0 & 0.5 & 1 & 1 & 0.29 & 2 & 2 & $1 ?$ & 5 & 5 & 0 & 0 & 0 & 6 & $>20$ & 0 & 0 \\
\hline I & $15 \mathrm{H}-3,32$ & 137.8 & $5 G Y 5 / 1$ & Clay & 5 & 0 & 0 & 1.2 & 0 & i & 0.29 & 2 & 2 & 0 & 4 & 4 & 0 & 0 & 0 & 6 & $>20$ & 2 & 0 \\
\hline 1 & $15 \mathrm{H}-3,145$ & 139.0 & Pale green & Calcareous clay & 6 & 0 & 0 & 9.9 & 2 & i & 0.50 & 0 & 0 & 3 & 4 & 6 & 0 & 0 & 0 & 4 & $>20$ & 2 & 0 \\
\hline i & $18 \mathrm{H}-1,13$ & 163.7 & Green gray & Clay & 4 & 0 & 0 & . & 0 & 2 & - & i & 1 & 0 & 5 & 5 & 0 & 0 & 0 & 6 & $>20$ & 0 & 0 \\
\hline I & $18 \mathrm{H}-3,145$ & 168.1 & Pale green & $\begin{array}{l}\text { Clayey nannofossil } \\
\text { ooze }\end{array}$ & - & . & . & 70.0 & 0 & $i$ & 0.27 & $:$ & : & - & 3 & 6 & 0 & 0 & 0 & 5 & $>20$ & 0 & 0 \\
\hline I & $19 X-3,46$ & 176.8 & White/green & $\begin{array}{l}\text { Clayey nannofossil } \\
\text { ooze }\end{array}$ & - & - & - & - & 0 & 0 & - & - & - & - & 2 & 4 & 0 & 0 & 0 & 6 & $>20$ & 0 & 0 \\
\hline II & $21 X-2,145$ & 195.7 & White/tan & Calcareous ooze & 3 & 0 & 0 & 80.9 & 2 & 2 & 0.22 & 0 & 0 & 1 & $2 ?$ & 5 & 6 & 3 & 0 & 3 & $>20$ & 2 & 0 \\
\hline II & $24 X-3,145$ & 226.3 & $\begin{array}{c}\text { White/tan } \\
\text { w/green }\end{array}$ & Foraminifera ooze & 4 & 0 & 0 & 89.1 & 4 & 4 & 0.07 & 0 & 0 & i & 2 ? & 5 & 6 & 3 & 0 & 3 & $>20$ & 2 & 0 \\
\hline II & $27 \mathrm{X}-1,41$ & 251.3 & $5 G Y 6 / 1$ & Calcareous ooze & 3 & 0 & 0 & 74.9 & 4 & 4 & 0.26 & 0 & 0 & 1 & 0 & 3 & 6 & 4 & 0 & 5 & $>20$ & 0 & 0 \\
\hline II & $27 \mathrm{X}-2,145$ & 253.9 & Brown/green & Calcareous ooze & 3 & 1 & 0 & 69.2 & 3 & 4 & 0.44 & 0 & 0 & 1 & $2 ?$ & 5 & 6 & 3 & 0 & 3 & $>20$ & 0 & 0 \\
\hline II & $31 X-3,140$ & 294.0 & Gray/green & Calcareous ooze & 4 & 0 & 0 & 79.8 & 4 & 4 & 0.14 & 0 & 0 & 2 & $2 ?$ & 6 & 5 & 3 & 0 & 4 & $>20$ & 0 & 0 \\
\hline II & $36 X-2,140$ & 340.8 & Green/white & Foraminifera ooze & 4 & 0 & 0 & 78.7 & 4 & 4 & 0.29 & 0 & 0 & 2 & $2 ?$ & 6 & 5 & 3 & 0 & 3 & $>20$ & 2 & 0 \\
\hline IIIIII & $39 \mathrm{X}-1,86$ & 367.6 & White/tan & Foraminifera ooze & 3 & 0 & 0 & 95.1 & 3 & 4 & 0.12 & 0 & 0 & 0 & $0 ?$ & 4 & 4 & 6 & 4 & 0 & . & 0 & 0 \\
\hline III & $4 \mathrm{R}-3,140$ & 383.4 & Gray & Calcareous ooze & 2 & 0 & 0 & 71.6 & 3 & 3 & 0.09 & 0 & 0 & 2 & 0 ? & 1 & 0 & 6 & 2 & 0 & . & 0 & 0 \\
\hline III & $7 R-4,75$ & 413.3 & Green & Calcareous ooze & 3 & 0 & 0 & 40.1 & 3 & 4 & 0.12 & 0 & 0 & 3 & $0 ?$ & 5 & 0 & 6 & 4 & 0 & - & 2 & 0 \\
\hline III & $10 \mathrm{R}-3,140$ & 440.9 & Gray & $\begin{array}{l}\text { Clayey nannofossil } \\
\text { ooze }\end{array}$ & 4 & 0 & 0 & 50.9 & 4 & 3 & 0.21 & 0 & 0 & 3 & $0 ?$ & 5 & 0 & 6 & 4 & 0 & . & 0 & 0 \\
\hline IV & IIR-I, 84 & 446.8 & $5 Y 4 / 1$ & Calcareous claystone & 4 & 0 & 0 & 32.9 & 0 & 3 & 0.31 & 2 & 0 & ? & 4 & 5 & 0 & 5 & 0 & 6 & - & 0 & 0 \\
\hline IV & $11 \mathrm{R}-1,119$ & 447.2 & $5 Y 6 / 1$ & $\begin{array}{l}\text { Clayey calcareous } \\
\text { ooze }\end{array}$ & 4 & 0 & 0 & 80.7 & 3 & 1 & 0.09 & i & 0 & 0 & 3 & 4 & 0 & 5 & 0 & 6 & 56 & 1 & 0 \\
\hline IV & $11 \mathrm{R}-4,61$ & 451.1 & $5 B 4 / 1$ & Claystone & 4 & 0 & 0 & 1.1 & 0 & 1 & 0.18 & 3 & 2 & 0 & 3 & 5 & 0 & 5 & 0 & 6 & 54 & 0 & 0 \\
\hline IV & $13 \mathrm{R}-2,61$ & 466.7 & $5 G Y 4 / 1$ & Claystone & 4 & 0 & 0 & 4.1 & 0 & i & 0.00 & 3 & 0 & 0 & 4 & 5 & 0 & 0 & 0 & 6 & 28 & 0 & 0 \\
\hline IV & $15 \mathrm{R}-1,115$ & 484.9 & Green & Claystone & 6 & 0 & 0 & 2.3 & 0 & 0 & 0.20 & 0 & 0 & 3 & 2 & 6 & 0 & 0 & 0 & 5 & 12 & 0 & 0 \\
\hline IV & $16 \mathrm{R}-3,48$ & 496.7 & - & Calcareous claystone & - & . & . & $\because$ & 0 & 0 & - & - & . & : & 0 & 5 & 0 & 0 & 0 & 6 & 12 & 0 & 0 \\
\hline IV & $17 \mathrm{R}-2,66$ & 504.6 & $5 Y R 6 / 6$ & $\begin{array}{l}\text { Clayey calcareous } \\
\text { ooze }\end{array}$ & 3 & 0 & 0 & 54.2 & 0 & 0 & 0.00 & 2 & 2 & $\mathrm{i}$ & 3 & 5 & 0 & 0 & 0 & 6 & 40 & 0 & 0 \\
\hline IV & $18 \mathrm{R}-3,140$ & 516.3 & Orange/brown & Calcareous claystone & 3 & 0 & 0 & 76.5 & 0 & 0 & 0.00 & 0 & 0 & 1 & 2 & 6 & 0 & 0 & 0 & 6 & - & 0 & 0 \\
\hline IV & $19 \mathrm{R}-3,77$ & 525.1 & Orange/brown & - & 3 & 0 & 0 & - & 0 & 0 & . & 0 & 0 & 0 & 0 & 1 & 0 & 0 & 0 & 6 & $<10$ & 0 & 2 \\
\hline IV & $20 \mathrm{R}-2, \quad 23$ & 532.5 & - & . & 6 & 0 & 0 & . & 0 & 0 & . & 4 & 4 & 0 & 0 & i & 0 & 0 & 0 & 6 & 10 & 0 & 0 \\
\hline IV & $22 \mathrm{R}-2, \quad 28$ & 551.9 & $5 G Y 5 / 1$ & Claystone & 4 & 0 & 0 & 2.4 & 0 & 1 & 0.04 & 4 & 0 & 0 & 3 & 0 & 0 & 0 & 0 & 6 & $<10$ & 0 & 3 \\
\hline IV & $22 \mathrm{R}-2,140$ & 553.0 & White/gray & Claystone & 3 & 0 & 0 & 68.6 & 0 & 0 & 0.00 & 0 & 0 & 0 & 3 & 0 & 0 & 0 & 0 & 6 & - & 0 & 3 \\
\hline $\mathrm{v}$ & $24 \mathrm{R}-1, \quad 92$ & 570.2 & $10 \mathrm{YR} 4 / 1$ & Claystone & 4 & 0 & 0 & 0.3 & 0 & 1 & 0.03 & 3 & 3 & 1 & 3 & 3 & 0 & 6 & 0 & 4 & 56 & 0 & 2 \\
\hline
\end{tabular}




\begin{tabular}{|c|c|c|c|c|c|c|c|c|c|c|c|c|c|c|c|c|c|c|c|c|c|c|c|}
\hline \multirow[b]{2}{*}{ M.A. } & \multirow[b]{2}{*}{ Core $(\mathrm{cm})$} & \multirow[b]{2}{*}{$\begin{array}{l}\text { Depth } \\
\text { (mbsf) }\end{array}$} & \multirow[b]{2}{*}{ Color } & \multirow[b]{2}{*}{ Lithology } & \multicolumn{3}{|c|}{ Silica } & \multicolumn{3}{|c|}{ Carbonate } & \multirow{2}{*}{$\begin{array}{c}\text { Org. } \\
\text { Ca } \\
\text { (wt\%) }\end{array}$} & \multicolumn{2}{|c|}{ Feldspar } & \multicolumn{10}{|c|}{ Clay minerals $(<2 \mu \mathrm{m})$} \\
\hline & & & & & Qtz & O-A & O-CT & $\begin{array}{l}\mathrm{CaCO}_{3} \\
(\%)\end{array}$ & Arag. & Dol. & & $\mathrm{K}$ & Plag & Mica & Illite & Kaol & Sep & Palyg & I/S/C & 1/S & $\% \mathrm{I}$ & Pyr & Zeol \\
\hline $\mathrm{v}$ & $24 \mathrm{R}-4,71$ & 574.5 & $5 \mathrm{YR} 3 / 1$ & Claystone & 5 & 0 & 0 & 0.7 & 0 & 1 & 0.05 & 0 & 0 & 1 & 3 & 4 & 0 & 5 & 0 & 6 & 52 & 0 & ? \\
\hline $\mathrm{v}$ & $25 \mathrm{R}-4,140$ & 584.9 & Brown/orange & Clayey chalk & . & . & . & 63.8 & 0 & 0 & 0.00 & - & - & . & 3 & 3 & 0 & $2(?)$ & 0 & 6 & . & 0 & 0 \\
\hline $\mathrm{v}$ & $26 \mathrm{R}-4,22$ & 593.0 & 5YR 4/4 & Claystone & 5 & 0 & 0 & 4.8 & 0 & 1 & 0.01 & 1 & 0 & 1 & 3 & 4 & 0 & 5 & 0 & 6 & 56 & 0 & 2 \\
\hline $\mathrm{v}$ & $27 \mathrm{R}-2, \quad 34$ & 599.3 & $5 Y R 4 / 1$ & Claystone & 4 & 0 & 0 & 0.3 & 0 & $i$ & 0.01 & 3 & $1(?)$ & $i$ & 4 & 3 & 0 & 5 & 0 & 6 & 50 & 0 & 2 \\
\hline $\mathrm{v}$ & $28 \mathrm{R}-1,55$ & 607.6 & SYR $3 / 1$ & Claystone & 4 & 0 & 0 & 0.2 & 0 & 0 & 0.01 & 2 & 2 & 1 & 0 & 0 & 0 & 5 & 0 & 6 & 40 & 0 & 0 \\
\hline $\mathrm{v}$ & $28 \mathrm{R}-2,140$ & 609.9 & Tan/black & Calcareous claystone & 5 & 0 & 0 & 16.2 & 0 & 0 & 0.00 & 0 & 0 & 2 & 3 & 3 & 0 & 4 & 0 & 6 & - & 0 & 0 \\
\hline $\mathrm{v}$ & $29 \mathrm{R}-3,76$ & 620.2 & $5 \mathrm{BG} 5 / 1$ & Claystone & 4 & 0 & 0 & 5.3 & 0 & 0 & 0.06 & 3 & 3 & 1 & 4 & 2 & 0 & 4 & 0 & 6 & 30 & 0 & \\
\hline $\mathrm{v}$ & $29 \mathrm{R}-5, \quad 2$ & 622.4 & Pale brown & Claystone & 6 & 0 & 1 ? & $\because$ & 0 & $1 ?$ & - & 4 & 2 & $i$ & 5 & 2 & 0 & 5 & 0 & 6 & 58 & $1 ?$ & 0 \\
\hline $\mathrm{v}$ & $29 \mathrm{R}-5,26$ & 622.7 & Pale brown & Claystone & 6 & 0 & 0 & . & 0 & $i$ & - & 4 & 4 & 3 & 5 & 4 & 0 & 5 & 0 & 6 & 51 & 0 & $2 ?$ \\
\hline $\mathrm{v}$ & $29 \mathrm{R}-5,70$ & 623.1 & Pale brown & Claystone & 6 & 0 & 0 & . & 0 & 1 & . & 4 & 4 & 3 & 0 & 0 & 0 & 5 & 0 & 6 & 47 & 0 & 2 \\
\hline $\mathrm{v}$ & $29 \mathrm{R}-5,124$ & 623.6 & Pale brown & Claystone & 6 & 0 & 0 & - & 0 & i & - & 3 & 3 & 1 & 0 & 0 & 0 & 5 & 0 & 6 & 43 & 0 & 2 \\
\hline $\mathrm{v}$ & $30 \mathrm{R}-4,98$ & 631.4 & $5 Y R 3 / 4$ & Claystone & 5 & 0 & 0 & 0.2 & 0 & 1 & 0.05 & 4 & 4 & 1 & 5 & 3 & 0 & 3 & 0 & 6 & 28 & 0 & 3 \\
\hline VI & $31 \mathrm{R}-2,130$ & 638.1 & Tan/brown & Calcareous claystone & 4 & 0 & 0 & 26.3 & 0 & 0 & 0.00 & 0 & 0 & 2 & 4 & 0 & 0 & 0 & 0 & 6 & - & 0 & 3 \\
\hline VI & $34 \mathrm{R}-3,115$ & 668.6 & Green & Calcareous claystone & 3 & 0 & 4 & 21.7 & 0 & 0 & 0.10 & 0 & 0 & 2 & 3 & 0 & 0 & 0 & 0 & 6 & . & 0 & 0 \\
\hline VI & 35R-2, 97 & 676.6 & Pale green & Calcareous claystone & 0 & 0 & 3 & - & 0 & 0 & - & 1 & 1 & 2 & 4 & 0 & 0 & 0 & 0 & 6 & $<10$ & 0 & 1 \\
\hline VI & 35R-3, 24 & 677.3 & Gray/black & Claystone & 5 & 0 & 4 & 0.3 & 0 & 0 & 0.13 & 2 & 2 & 2 & 5 & 1 & 0 & 0 & 0 & 6 & $<10$ & 1 & 0 \\
\hline VI & $35 \mathrm{R}-3,86$ & 678.0 & $2.5 \mathrm{Y} 2 / 0$ & Calcareous siltstone & 3 & 0 & 6 & 4.7 & 0 & 0 & 0.67 & $i$ & 1 & $i$ & 1 & i & 0 & 0 & 0 & 6 & $<10$ & i & 0 \\
\hline VI & $37 \mathrm{R}-3,40$ & 696.4 & $5 Y 5 / 2$ & Calcareous claystone & 0 & 0 & 2 & 34.7 & 0 & 1 ? & 0.06 & 2 & 2 & $i$ & 3 & $i$ & 0 & 0 & 0 & 6 & $<10$ & 0 & 0 \\
\hline VI & $37 \mathrm{R}-3,140$ & 697.4 & Tan & $\begin{array}{l}\text { Clayey chalk } \\
\text {. }\end{array}$ & 0 & 0 & 1 & 51.9 & 0 & 0 & 0.15 & 0 & 0 & 2 & 3 & 0 & 0 & 0 & 0 & 6 & $<10$ & 2 & 0 \\
\hline VI & $39 \mathrm{R}-3,80$ & 715.5 & Pale green & Clayey chalk & 4 & 0 & 3 & - & 0 & 0 & - & 0 & 0 & 2 & 3 & 0 & 0 & 0 & 0 & 6 & $<10$ & 0 & 0 \\
\hline VI & $39 \mathrm{R}-3,101$ & 715.7 & $5 Y 4 / 1$ & Calcareous claystone & 5 & 0 & 1 & 1.0 & 0 & 0 & 0.04 & 2 & 2 & 2 & 5 & 1 & 0 & 0 & 0 & 6 & $<10$ & 2 & 3 \\
\hline VI & $39 \mathrm{R}-3,140$ & 716.1 & Tan & Calcareous claystone & 4 & 0 & 5 & 20.6 & 0 & 0 & 0.00 & 0 & 0 & 2 & 3 & 0 & 0 & 0 & 0 & 6 & - & 2 & 0 \\
\hline VI & $40 R-3,104$ & 724.9 & $5 Y 2.5 / 1$ & Claystone & 6 & 0 & 1 & 0.5 & 0 & 0 & 0.22 & 3 & 3 & 2 & 5 & 1 & 0 & 0 & 0 & 6 & $<10$ & $2(?)$ & 0 \\
\hline VI & $42 \mathrm{R}-2,41$ & 741.7 & $5 \mathrm{G} 4 / 1$ & Claystone & 6 & 0 & $1 ?$ & 0.6 & 0 & 1 & 0.15 & 1 & 1 & 0 & 3 & 1 & 0 & 0 & 0 & 6 & $<10$ & 0 & 0 \\
\hline VI & $42 \mathrm{R}-3,140$ & 744.2 & Green & Claystone & 6 & 0 & 0 & 0.6 & 0 & 0 & 0.08 & 0 & 0 & 3 & 3 & 1 & 0 & 0 & 0 & 6 & $<10$ & 2 & 0 \\
\hline VI & $44 \mathrm{R}-2, \quad 38$ & 760.4 & Olive gray & Claystone & 5 & 0 & 0 & - & 0 & 3 & - & 2 & 2 & 0 & 4 & 3 & 0 & 0 & 0 & 6 & $<10$ & 0 & 0 \\
\hline VI & $44 \mathrm{R}-2,73$ & 760.7 & Olive gray & Claystone & 6 & 0 & 0 & . & 0 & 0 & - & 3 & 3 & 1 & 4 & 2 & 0 & 0 & 0 & 6 & $<10$ & 1 & 0 \\
\hline vI & $44 \mathrm{R}-2,95$ & 761.0 & Olive gray & Claystone & 6 & 0 & 0 & . & 0 & 1 & . & 3 & 3 & i & 4 & 2 & 0 & 0 & 0 & 6 & $<10$ & $i$ & 0 \\
\hline VI & $44 \mathrm{R}-2,119$ & 761.2 & Olive gray & Claystone & 6 & 0 & 0 & . & 0 & 0 & . & 3 & 3 & 1 & 4 & 2 & 0 & 0 & 0 & 6 & $<10$ & 1 & 0 \\
\hline VI & $44 \mathrm{R}-4,55$ & 763.6 & $5 Y 3 / 1$ & Claystone & 6 & 0 & 0 & 1.1 & 0 & 0 & 0.29 & 3 & 3 & 1 & 4 & 2 & 0 & 0 & 0 & 6 & $<10$ & 1 & 0 \\
\hline VI & $45 \mathrm{R}-2,70$ & 770.4 & $5 \mathrm{R} 3 / 2$ & Claystone & 6 & 0 & 0 & 0.7 & 0 & 1 & 0.09 & 3 & 2 & 0 & 4 & 2 & 0 & 0 & 0 & 6 & 10 & 0 & 0 \\
\hline vi & $45 \mathrm{R}-3,140$ & 772.6 & Red/brown & Claystone & 6 & 0 & 0 & 3.6 & 0 & 0 & 0.14 & 0 & 0 & 3 & 3 & 0 & 0 & 0 & 0 & 6 & . & 0 & 0 \\
\hline vI & 48R-3, 140 & 801.3 & Brown & Claystone & 6 & 0 & 0 & 1.0 & 0 & 0 & 0.40 & 0 & 0 & 3 & 3 & 0 & 0 & 0 & 0 & 6 & . & 2 & 0 \\
\hline vi & S0R-1,110 & 816.7 & $2.5 \mathrm{YR} 3 / 2$ & Claystone & 6 & 0 & 0 & 0.6 & 0 & 0 & 0.01 & 1 & 1 & $1(?)$ & 4 & 1 & 0 & 0 & 0 & 6 & 10 & 2 & 0 \\
\hline vi & $51 \mathrm{R}-1,84$ & 825.8 & $2.5 \mathrm{Y} \mathrm{N} 3$ & Claystone & 6 & 0 & 0 & 0.4 & 0 & 0 & 0.18 & 3 & 3 & $i$ & 4 & 2 & 0 & 0 & 0 & 6 & 10 & 1 & 0 \\
\hline VI & $51 \mathrm{R}-3,140$ & 829.4 & Brown/green & Claystone & 6 & 0 & 0 & 1.4 & 0 & 0 & 0.00 & 0 & 0 & 2 & 3 & 0 & 0 & 0 & 0 & 6 & 10 & 0 & 0 \\
\hline VI & $54 \mathrm{R}-3,140$ & 857.9 & Tan/brown & "Chert,claystone" & 6 & 0 & 0 & 0.3 & 0 & 0 & 0.14 & 0 & 0 & 2 & 3 & 0 & 0 & 0 & 0 & 6 & . & 0 & 0 \\
\hline VI & $56 \mathrm{R}-4,148$ & 878.5 & Tan & Calcareous claystone & 3 & 0 & 0 & - & 0 & 0 & - & $1(?)$ & 0 & i & 2 & 0 & 0 & 0 & 0 & 6 & 12 & 0 & 0 \\
\hline VI & $57 \mathrm{R}-3,140$ & 886.1 & White/gray & $\begin{array}{l}\text { Nannofossil } \\
\text { claystone }\end{array}$ & 6 & 0 & 0 & 28.2 & 0 & 0 & 0.07 & 0 & 0 & 2 & 3 & 0 & 0 & 0 & 0 & 6 & 12. & 2 & 0 \\
\hline VI & $57 \mathrm{R}-4, \quad 72$ & 886.9 & Tan & Calcareous claystone & 4 & 0 & 0 & 9.0 & 0 & 0 & 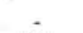 & 1 & 0 & 2 & 1 & 1 & 0 & 0 & 0 & 6 & 10 & 0 & 0 \\
\hline VI & $58 \mathrm{R}-4,67$ & 896.4 & 2.5 YR $2.5 / 4$ & $\begin{array}{l}\text { Claystone } \\
\text { Sagtoin }\end{array}$ & 6 & 0 & 0 & 0.3 & 0 & 1 & 0.01 & 1 & 1 & 2 & 2 & 0 & 0 & 0 & 0 & 6 & 30 & 1 & 0 \\
\hline VII & $60 \mathrm{R}-3,140$ & 911.9 & Red/brown & Claystone & 6 & 0 & 0 & 0.4 & 0 & 0 & 0.02 & 0 & 0 & 3 & 3 & 0 & 0 & 0 & 0 & 6 & 17 & 0 & 0 \\
\hline VII & $60 \mathrm{R}-5,120$ & 914.7 & 2.5 YR $3 / 6$ & Claystone & 3 & 0 & 0 & 0.3 & 0 & 1 & 0.09 & 4 & 0 & 2 & 0 & 0 & 0 & 0 & 0 & 6 & 17 & 1 & 0 \\
\hline VII & $61 \mathrm{R}-5,81$ & 923.7 & $5 Y R 3 / 2$ & Calcareous claystone & 4 & 0 & 0 & 27.3 & 0 & $i$ & 0.00 & 3 & 0 & $i$ & 3 & 0 & 0 & 0 & 0 & 6 & 40 & 2 & 0 \\
\hline VII & $62 \mathrm{R}-3,73$ & 930.1 & Mud brown & Claystone & 6 & 0 & 0 & $\because$ & 0 & i & - & 2 & 2 & $i$ & 3 & 0 & 0 & 0 & 0 & 6 & 33 & 1 & 0 \\
\hline VII & $62 \mathrm{R}-3,80$ & 930.2 & SYR $3 / 2$ & Claystone & 6 & 0 & 0 & 0.5 & 0 & 1 & 0.00 & 3 & 0 & 2 & 4 & 0 & 0 & 0 & 0 & 6 & 33 & 1 & 0 \\
\hline
\end{tabular}

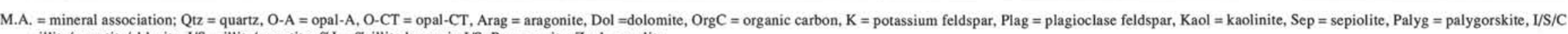

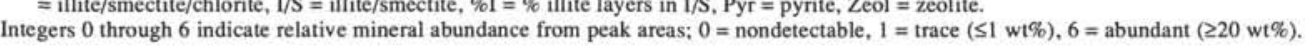




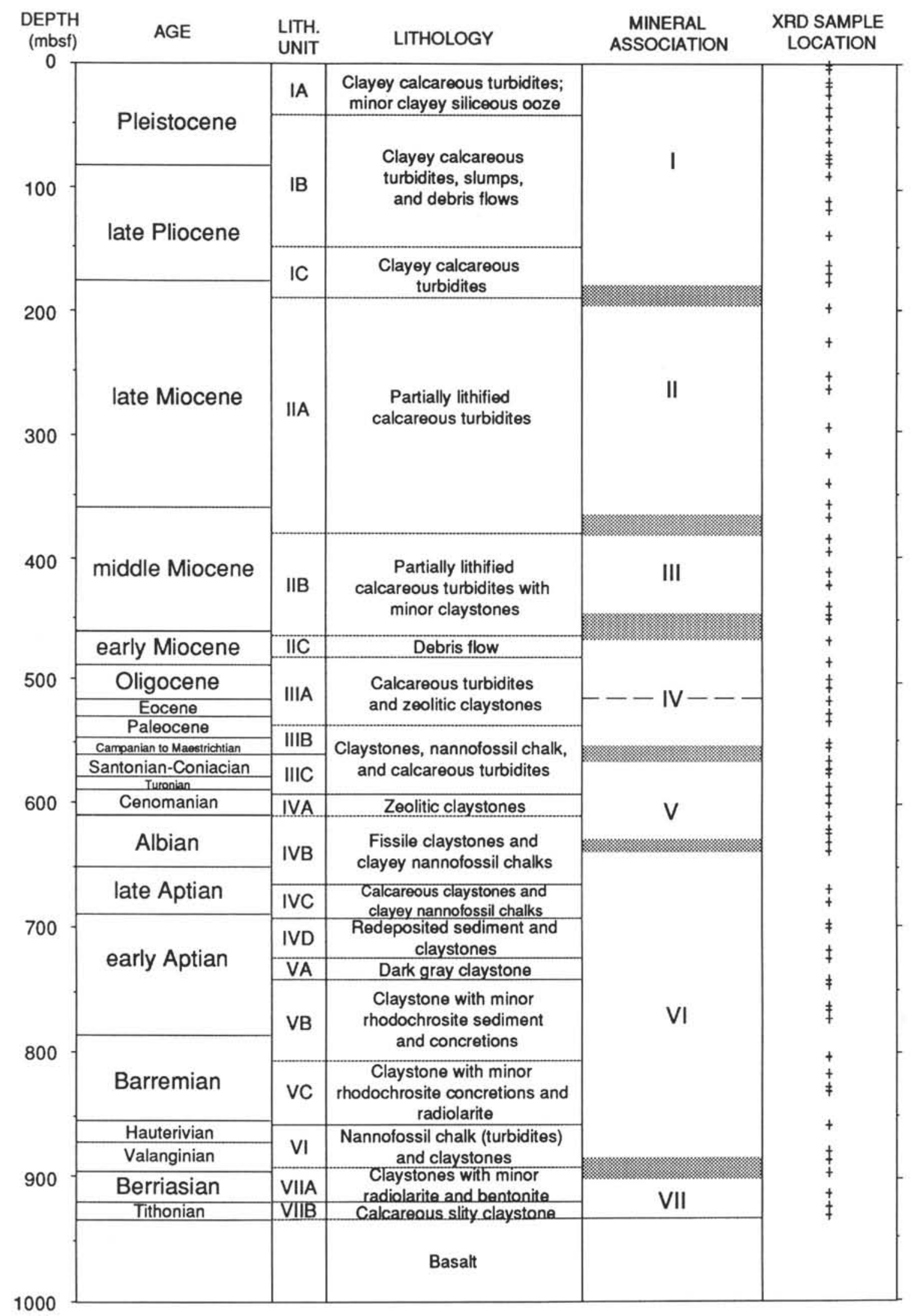

Figure 2. Correlation of mineral associations defined at Site 765 with sample location, sediment depth, age, lithology, and lithologic units. Stippled areas denote transition between mineral associations. 
$\AA$

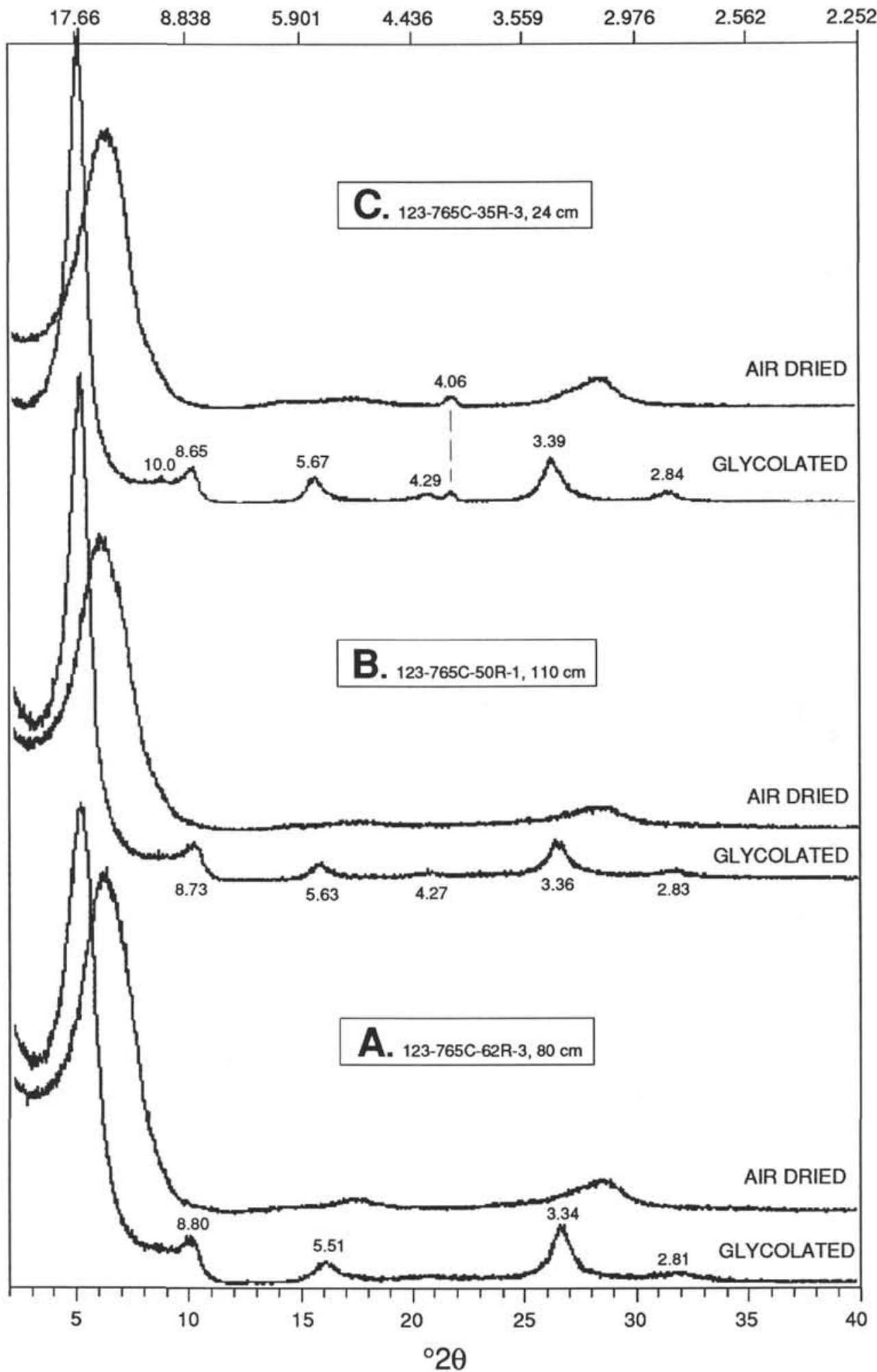

Figure 3. A. Air-dried and glycolated XRD patterns of the $<0.5-\mu \mathrm{m}$ size fraction of Sample 123-765C-62R-3, $80 \mathrm{~cm}$, from mineral Association VII that contains mixed-layered I/S with approximately $33 \%$ illite layers. B. Air-dried and glycolated XRD patterns of the $<0.5-\mu \mathrm{m}$ size fraction of Sample $123-765 \mathrm{C}-50 \mathrm{R}-1,110 \mathrm{~cm}$, from mineral Association VI that contains mixed-layered I/S with approximately $10 \%$ illite layers. C. Air-dried and glycolated XRD patterns of the $<0.5-\mu \mathrm{m}$ size fraction of Sample 123-765C-35R-39, $24 \mathrm{~cm}$, from mineral Association VI that contains highly smectitic mixed-layered I/S ( $<10 \%$ illite layers) as well as minor amounts of discrete illite ( $10 \AA$ peak) and opal-CT $(4.06 \AA$ peak). 


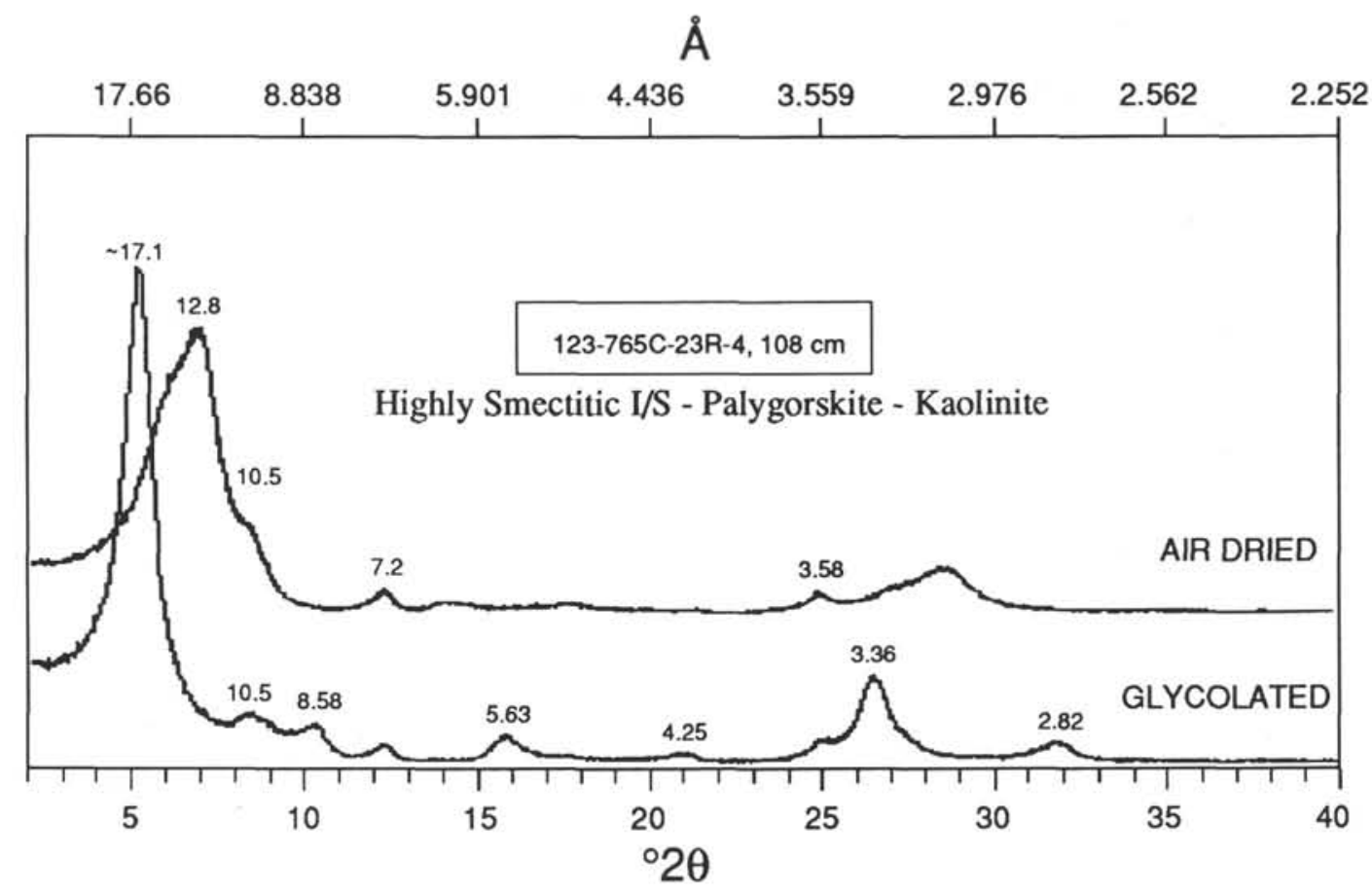

Figure 4. Air-dried and glycolated XRD patterns of the $<0.5-\mu \mathrm{m}$ size fraction of Sample $123-765 \mathrm{C}-23 \mathrm{R}-4,108 \mathrm{~cm}$. This sample is transitional between mineral Association $\mathrm{V}$ and the lower part of mineral Association IV and contains a mixture of highly smectitic mixed-layered I/S (about $10 \%$ illite layers; glycolated $17.1,8.58,5.63,4.25,3.36$, and $2.82 \AA$

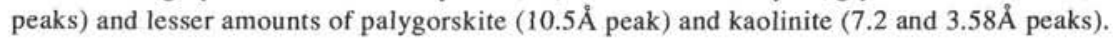

than $10 \%$ interstratified illite layers. The $\mathrm{I} / \mathrm{S}$ in the samples analyzed contain a large amount of $\mathrm{Fe}$ and $\mathrm{Mg}$ relative to $\mathrm{Al}$ in the octahedral layer. The Fe content of the clay minerals may be lower than that shown in Table 3, if our assumption of negligible $\mathrm{Fe}$ oxide coatings is incorrect. However, the presence of pyrite and the green, as opposed to red or brown, color of several of the samples analyzed suggest that Fe oxides are not abundant.

\section{ORIGIN OF THE CLAY MINERALS}

The clay minerals from Site 765 can be divided into (1) detrital clay minerals that include mixed-layered I/S with a large and variable percentage of illite layers, kaolinite, discrete illite, and mica; and (2) potentially diagenetic/authigenic clay minerals that include highly smectitic I/S, I/S that has undergone illitization, sepiolite, palygorskite, and saponite or mixed-layered I/S/C.

\section{Detrital Clay Minerals}

Detrital minerals that occur in the $<2-\mu \mathrm{m}$ size fraction include $\mathrm{I} / \mathrm{S}$ with variable amounts of more than $10 \%$ randomly interstratified illite layers, kaolinite, discrete illite, and mica (Table 1). Kaolinite, illite, and especially mica tend to occur in the coarse clay- to fine silt-size fraction ( 1 to $38 \mu \mathrm{m}$ ), whereas the I/S occurs mostly in the $<1-\mu \mathrm{m}$ size fraction. Mica minerals are considered separate from illite here and generally include muscovite and biotite. I/S, kaolinite, illite, and mica are fairly ubiquitous at Site 765; only the basal mineral Associations VI and VII contain trace-to-nondetectable amounts of kaolinite. The kaolinite content tends to increase from the base of the section up to the Neogene (485 mbsf) and then remains fairly abundant in the Neogene and Pleistocene (Fig. 7). The abundance of illite in mineral Associations II and III is uncertain because the $10 \AA$ illite peak was difficult to differentiate from the shoulder of the large palygorskite peak at $10.5 \AA$. Detrital I/S occurs in mineral Asso- ciations I, II, IV (upper part), V, and possibly VII. The I/S in these mineral associations is considered detrital because authigenic I/S has been predicted to have $<10 \%$ illite layers, given the low grade of burial diagenesis at Site 765 (see below). The highly variable amounts of interstratified illite in the I/S of these mineral associations is consistent with multiple detrital sources.

Detrital clay minerals, along with silt-sized grains of quartz and feldspar, were derived from weathering of adjacent continental landmasses. Terrigenous silt and clay were transported out onto the Australian continental margin as suspended material carried by rivers or wind. This terrigenous material was then transported from the continental margin to the Argo Basin by gravity flows, such as turbidity currents and debris flows (Ludden, Gradstein, et al., 1990). In addition, some of the detrital minerals may have been transported by high-altitude winds.

Kaolinite is usually indicative of low-latitude source areas (Biscaye, 1965), where kaolinite forms as an alteration product during the intense weathering of feldspar minerals. The change in the paleolatitude of Site 765 from approximately $40^{\circ}$ to $17^{\circ} \mathrm{S}$ (Ludden, Gradstein, et al., 1990) corresponds to an increase in the kaolinite content during the Late Cretaceous (Fig. 7). Prior to the Late Cretaceous, the paleolatitude of Site 765 may have been too far south to have received significant amounts of kaolinite. In addition, terrigenous kaolinite may have been diluted by a greater influx of locally derived volcaniclastics during the Early Cretaceous (see below). The high kaolinite content of surface sediments off the coast of western Australia was derived from the extensive kaolinite-rich laterite deposits in the desert regions of western Australia (Kennett, 1982). Dust storms in the arid regions of western Australia may have been an important transport mechanism of detrital minerals such as kaolinite. Nearly all of the clay minerals from the uppermost Pliocene and Pleistocene sediments at Site 765 (mineral Association I) appear to be detrital. 
$\AA$

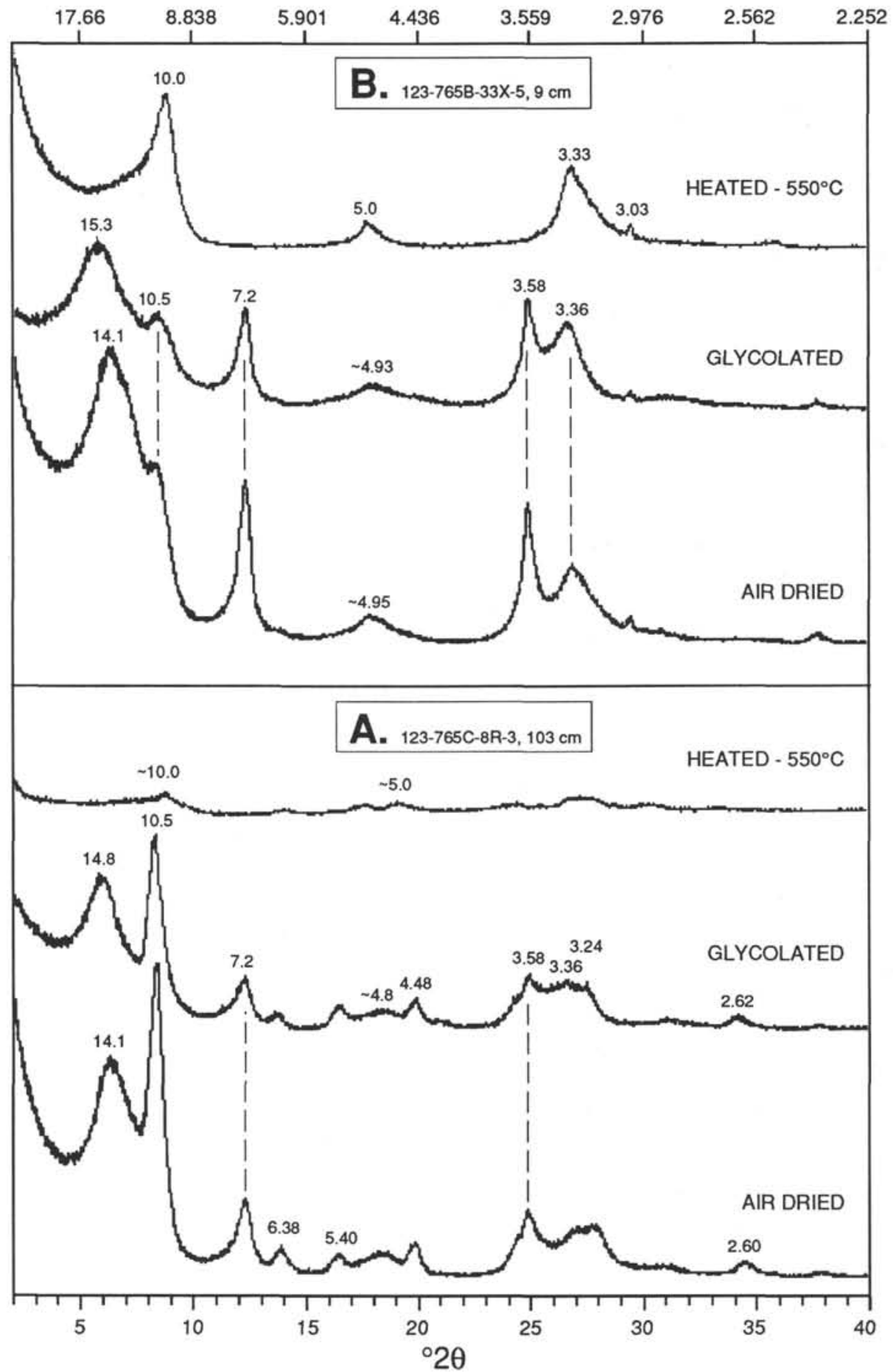

Figure 5. A. Air-dried, glycolated, and heated patterns of the $<0.5-\mu \mathrm{m}$ size fraction of Sample 123-765C-8R-3, $103 \mathrm{~cm}$, from mineral Association III. This sample contains palygorskite (10.5, $6.38,5.40,4.48$, and $3.24 \AA$ peaks) and kaolinite ( 7.2 and $3.58 \AA$ peaks), and saponite or mixed-layered I/S/C. B. Air-dried, glycolated, and heated patterns of the $<0.5-\mu \mathrm{m}$ size fraction of Sample 123-765B-33X-5, $9 \mathrm{~cm}$. This sample is from mineral Association II, but its XRD patterns are more

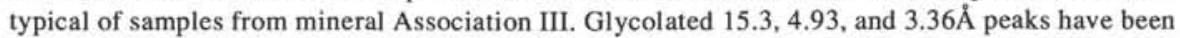
tentatively interpreted as either a mixed-layered $\mathrm{I} / \mathrm{S} / \mathrm{C}$ or a saponite that has resisted complete glycolation. Palygorskite ( $10.5 \AA$ peak), kaolinite (7.2 and $3.58 \AA$ peaks), and calcite ( $3.03 \AA$ peak) 


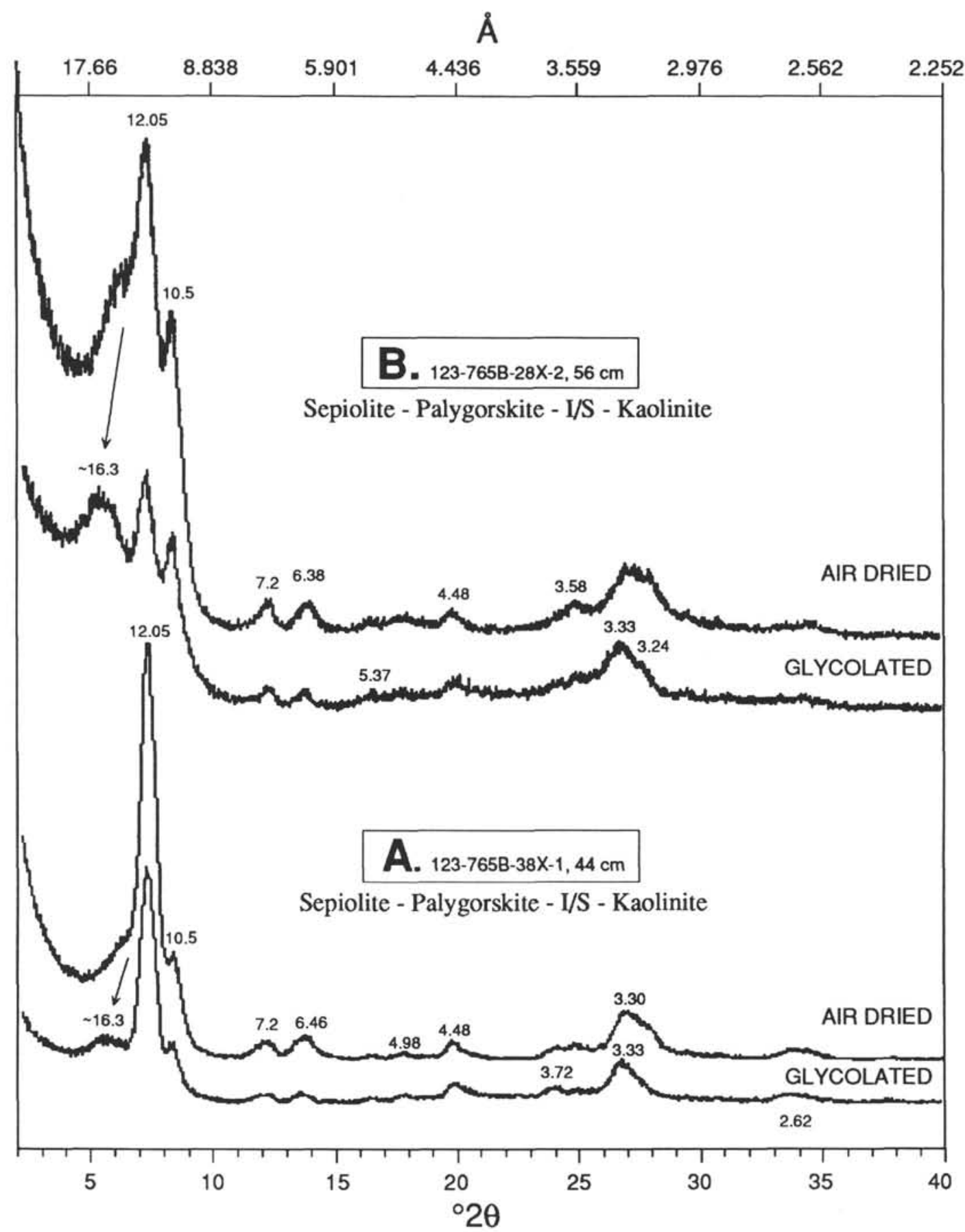

Figure 6. A. Air-dried and glycolated XRD patterns of the $<0.5-\mu \mathrm{m}$ size fraction of Sample 123-765B-38X-1, $44 \mathrm{~cm}$, from mineral Association II. This sample is dominated by sepiolite (12.05A peak), but also contains mixed-layered $\mathrm{I} / \mathrm{S}$, palygorskite, and kaolinite. B. Air-dried and glycolated XRD patterns of the $<0.5-\mu \mathrm{m}$ size fraction of Sample 123-765B-28X-2, $56 \mathrm{~cm}$, from mineral Association II. This sample contains sepiolite, palygorskite, kaolinite, and an expandable mixed-layered $\mathrm{I} / \mathrm{S}$.

\section{Diagenetic Clay Minerals}

\section{Illite/Smectite}

Highly smectitic I/S at Site 765 that contains less than $10 \%$ illite layers has been interpreted as derived primarily from the alteration of volcanic material. This interpretation is supported by the occurrence of bentonite layers and zeolite minerals in the sediment intervals containing highly smectitic I/S (Fig. 7). The bentonite layers were originally volcanic ash deposits that have been altered to nearly $100 \%$ smectite clay minerals (Grim and Güven, 1978). Bentonite layers recovered from the Exmouth Plateau and Argo Basin were derived from the alteration of dacitic to rhyolitic ash (von Rad and Thurow, in press). A volcanic origin for the clay minerals in the Lower to mid-Cretaceous sediments at Site 765 is consistent with the increase in volcanic activity expected during the early formation of oceanic crust in the Argo 
Table 2. Approximate chemical and mineral composition of clay-size separates from Hole $765 \mathrm{C}$.

\begin{tabular}{|c|c|c|c|c|c|c|c|c|c|c|c|c|c|c|}
\hline \multirow{2}{*}{$\begin{array}{l}\text { Core, sect., } \\
\text { interval }(\mathrm{cm})\end{array}$} & \multirow{2}{*}{$\begin{array}{l}\text { Depth } \\
\text { (mbsf) }\end{array}$} & \multicolumn{13}{|c|}{ Major oxides (wt\%) } \\
\hline & & $\mathrm{SiO}_{2}$ & $\mathrm{TiO}_{2}$ & $\mathrm{Al}_{2} \mathrm{O}_{3}$ & $\mathrm{Fe}_{2} \mathrm{O}_{3}$ & $\mathrm{MnO}$ & $\mathrm{MgO}$ & $\mathrm{CaO}$ & $\mathrm{Na}_{2} \mathrm{O}$ & $\mathrm{K}_{2} \mathrm{O}$ & $\mathrm{P}_{2} \mathrm{O}_{5}$ & Total & Mineralogy & LOI \\
\hline \multirow[t]{7}{*}{$7 R-4,75-85$} & \multirow[t]{7}{*}{413.3} & 52.61 & 0.70 & 16.74 & 5.81 & 0.07 & 8.50 & 12.19 & 0.26 & 1.71 & 0.05 & 98.63 & & 17.9 \\
\hline & & 28.30 & & 3.00 & 4.41 & & 4.74 & & & & & 40.4 & Palygorskite & \\
\hline & & 6.02 & & 3.20 & 0.55 & & 0.33 & 0.08 & & 0.85 & & 11.0 & Illite & \\
\hline & & 9.42 & & 8.00 & & & & & & & & 17.4 & Kaolinite & \\
\hline & & & & & & & & & & & & 0.0 & Quartz & \\
\hline & & 8.87 & 0.70 & 2.54 & 0.85 & 0.07 & 3.43 & 2.09 & 0.26 & 0.86 & 0.05 & 19.7 & I/S/Chl & \\
\hline & & & & & & & & 10.02 & & & & 17.9 & Calcite & \\
\hline \multirow[t]{6}{*}{$15 \mathrm{R}-\mathrm{I}, 115-125$} & \multirow[t]{6}{*}{484.9} & 59.68 & 1.06 & 21.15 & 8.63 & 0.04 & 3.18 & 0.59 & 2.18 & 1.87 & 0.06 & 98.4 & & 10.5 \\
\hline & & 6.54 & & 3.48 & 0.60 & & 0.36 & 0.08 & & 0.93 & & 12.0 & Illite & \\
\hline & & 10.00 & & 8.50 & & & & & & & & 18.5 & Kaolinite & \\
\hline & & 5.14 & & & & & & & & & & 5.1 & Quartz & \\
\hline & & 38.00 & 1.06 & 9.17 & 8.03 & 0.04 & 2.82 & 0.23 & 2.18 & 0.94 & 0.06 & 62.5 & $\mathrm{I} / \mathrm{S}$ & \\
\hline & & & & & & & & 0.28 & & & & 0.5 & Calcite & \\
\hline \multirow[t]{6}{*}{$28 \mathrm{R}-2,140-150$} & \multirow[t]{6}{*}{610} & 56.99 & 0.96 & 16.85 & 10.26 & 0.56 & 3.93 & 5.57 & 1.20 & 3.28 & 0.11 & 99.7 & & 9.7 \\
\hline & & 11.61 & & 6.17 & 1.06 & & 0.63 & 0.15 & & 1.64 & & 21.3 & Illite & \\
\hline & & 2.94 & & 2.50 & & & & & & & & 5.4 & Kaolinite & \\
\hline & & 4.44 & & & & & & & & & & 4.4 & Quartz & \\
\hline & & 38.00 & 0.96 & 8.18 & 9.20 & 0.56 & 3.30 & 1.11 & 1.20 & 1.64 & 0.11 & 64.3 & $\mathrm{I} / \mathrm{S}$ & \\
\hline & & & & & & & & 4.31 & & & & 7.7 & Calcite & \\
\hline \multirow[t]{5}{*}{$42 \mathrm{R}-3,140-150$} & \multirow[t]{5}{*}{744.3} & 68.18 & 0.80 & 14.15 & 9.01 & 0.05 & 3.35 & 0.73 & 1.01 & 2.41 & 0.05 & 99.7 & & 6.0 \\
\hline & & 8.50 & & 4.52 & 0.78 & & 0.47 & 0.11 & & 1.20 & & 15.6 & Illite & \\
\hline & & 16.68 & & & & & & & & & & 16.7 & Quartz & \\
\hline & & 43.00 & 0.80 & 9.63 & 8.23 & 0.05 & 2.88 & 0.45 & 1.01 & 1.21 & 0.05 & 67.3 & I/S & \\
\hline & & & & & & & & 0.17 & & & & 0.3 & Calcite & \\
\hline \multirow[t]{5}{*}{$48 \mathrm{R}-3,140-150$} & \multirow[t]{5}{*}{801.4} & 67.97 & 0.94 & 11.62 & 10.57 & 0.08 & 3.24 & 1.42 & 1.00 & 2.04 & 0.45 & 99.3 & & 5.8 \\
\hline & & 7.22 & & 3.84 & 0.66 & & 0.40 & 0.09 & & 1.02 & & 13.2 & Illite & \\
\hline & & 18.75 & & & & & & & & & & 18.8 & Quartz & \\
\hline & & 42.00 & 0.94 & 7.78 & 9.91 & 0.08 & 2.84 & 1.05 & 1.00 & 1.02 & 0.45 & 67.1 & $\mathrm{I} / \mathrm{S}$ & \\
\hline & & & & & & & & 0.28 & & & & 0.5 & Calcite & \\
\hline \multirow[t]{5}{*}{$60 \mathrm{R}-3,140-150$} & \multirow[t]{5}{*}{912.0} & 65.78 & 0.63 & 14.43 & 8.43 & 0.19 & 4.00 & 1.12 & 2.79 & 1.70 & 0.19 & 99.3 & & 8.4 \\
\hline & & 6.03 & & 3.20 & 0.55 & & 0.33 & 0.08 & & 0.85 & & 11.0 & Illite & \\
\hline & & 10.25 & & & & & & & & & & 10.2 & Quartz & \\
\hline & & 49.50 & 0.63 & 11.23 & 7.88 & 0.19 & 3.67 & 0.93 & 2.79 & 0.85 & 0.19 & 77.9 & I/S & \\
\hline & & & & & & & & 0.11 & & & & 0.2 & Calcite & \\
\hline
\end{tabular}

LOI $=$ loss on ignition.

Table 3. Structural formulas of mixed-layer illite/smectite from Hole $765 \mathrm{C}$.

\begin{tabular}{lccccc}
\hline $\begin{array}{l}\text { Core/section: } \\
\text { Interval (cm): }\end{array}$ & $\begin{array}{c}15 \mathrm{R}-1 \\
115-125\end{array}$ & $\begin{array}{c}28 \mathrm{R}-2 \\
140-150\end{array}$ & $\begin{array}{c}42 \mathrm{R}-3 \\
140-150\end{array}$ & $\begin{array}{c}48 \mathrm{R}-3 \\
140-150\end{array}$ & $\begin{array}{c}60 \mathrm{R}-3 \\
140-150\end{array}$ \\
\hline Tetrahedral & & & & & \\
$\mathrm{Si}$ & & & & & \\
$\mathrm{Al}$ & 3.80 & 3.78 & 3.94 & 3.94 & 3.93 \\
& 0.20 & 0.22 & 0.06 & 0.06 & 0.07 \\
Octahedral & & & & & \\
& & & & & \\
Al & 0.88 & 0.74 & 0.98 & 0.80 & 0.98 \\
Fe (total) & 0.60 & 0.69 & 0.57 & 0.70 & 0.47 \\
Mg & 0.42 & 0.49 & 0.39 & 0.40 & 0.43 \\
Ti & 0.08 & 0.07 & 0.06 & 0.07 & 0.04 \\
Sum & 1.98 & 1.99 & 2.00 & 1.97 & 1.92 \\
Charge & -0.40 & -0.45 & -0.33 & -0.42 & -0.63 \\
Total layer charge & -0.60 & -0.67 & -0.39 & -0.48 & -0.70 \\
& & & & & \\
Exchangeable & & & & & \\
K & & & & & \\
Na & 0.12 & 0.21 & 0.14 & 0.12 & 0.09 \\
Ca & 0.42 & 0.23 & 0.18 & 0.18 & 0.43 \\
Interlayer charge & 0.02 & 0.12 & 0.04 & 0.11 & 0.08 \\
& 0.58 & 0.68 & 0.40 & 0.52 & 0.68
\end{tabular}

Basin. The elemental composition of the bulk Lower Cretaceous sediments is similar to post-Archean sedimentary rocks from the western Australian continent and suggests that aluminosilicate minerals at Site 765 may have been derived from these continental basins (Plank and Ludden, this volume). Therefore, Cretaceous sediments at Site 765 probably consist of both volcaniclastic and terrigenous material, but the highly smectitic composition of the I/S suggests that volcaniclastic sediments were generally most abundant during the Early Cretaceous.

The association of highly smectitic I/S, zeolite minerals, and bentonite layers in Paleocene and Eocene sediments at Site 765 (lower portion of mineral Association IV) suggests that this was a period of increased volcanism in this area. Ash falls from the more recent volcanic activity associated with the Indonesian Arc andesites do not appear to have reached Site 765 (Ninkovich, 1979; Plank and Ludden, this volume). This is supported by the absence of highly smectitic I/S, zeolite minerals, or discrete ash layers in Pliocene and Pleistocene sediments at Site 765.

The alteration of volcanic glass to smectite occurs rapidly, compared to the illitization of smectite (Eberl and Hower, 1976). Studies of the Miocene Monterey Formation indicate that the alteration of silicic ash to smectite results in the loss of potassium and silica and the uptake of magnesium and calcium (Bramlette, 1946; Compton, in press). The alteration of ash to smectite was found to coincide approximately with the transformation of biogenic (opal-A) silica to opal-CT in the Monterey Formation. Bentonite layers occur in opal-A and opal-CT sediments, and 


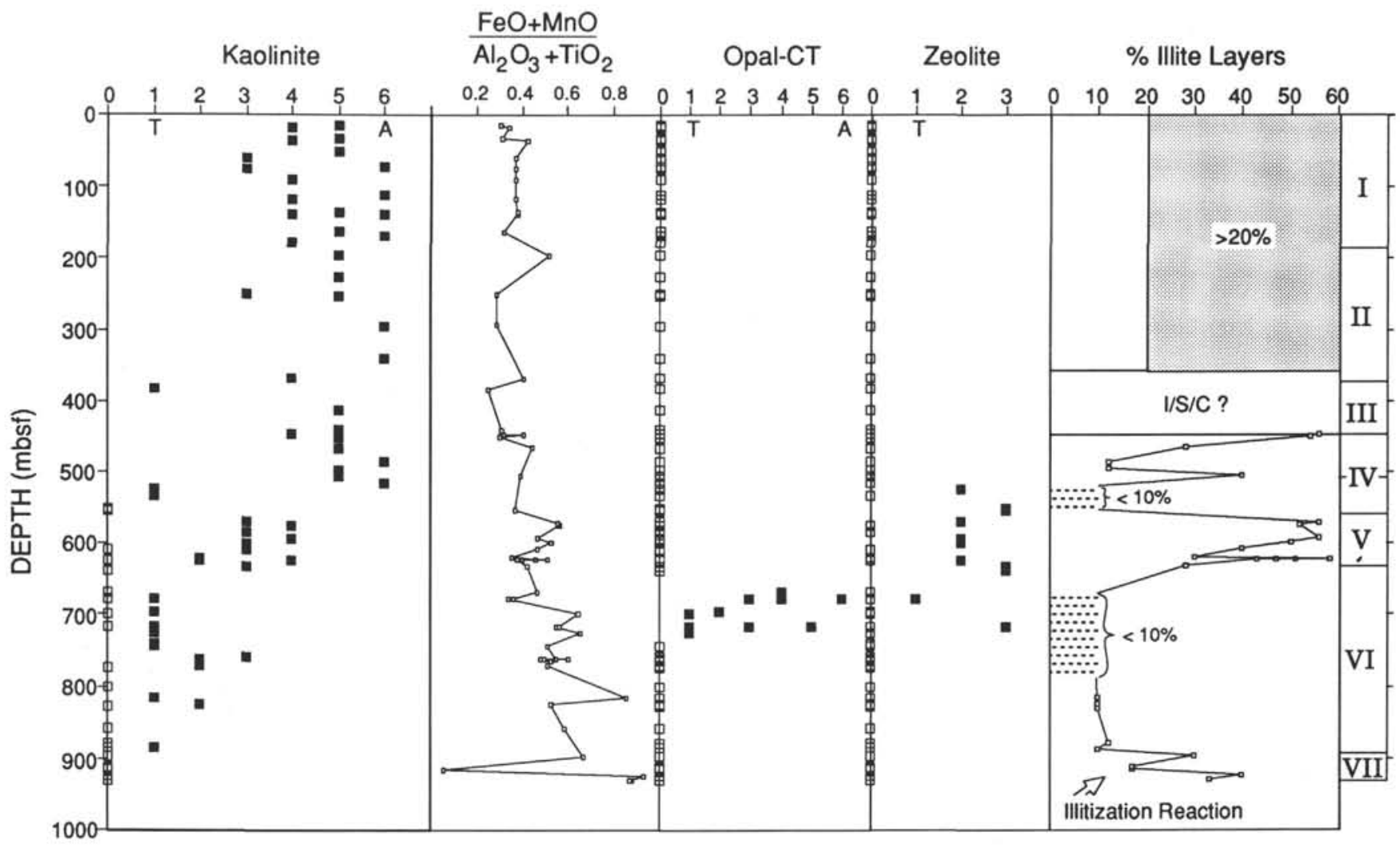

Figure 7. Relative abundances $(0=$ nondetectable, $1=$ trace $(\mathrm{T},<1 \mathrm{wt} \%)$ to $6=$ abundant $(\mathrm{A},>20 \mathrm{wt} \%)$ of kaolinite, opal-CT, and zeolite; the $(\mathrm{FeO}+$ $\mathrm{MnO}) /\left(\mathrm{Al}_{2} \mathrm{O}_{3}+\mathrm{TiO}_{2}\right)$ ratio of the bulk sediment; and the percentage of illite layers in the mixed-layered $\mathrm{I} / \mathrm{S}$ as a function of sediment depth at Site 765. Mineral Associations I through VII are shown on the far right.

metabentonites (see below) occur in diagenetic quartz-bearing sediments at Site 765. Basalt pebbles were observed in the Lower to mid-Cretaceous sediments at Site 765 and are thought to have been eroded from local exposures of mid-ocean-ridge basalt during rapid initial subsidence of the Argo Basin (Ludden, Gradstein, et al., 1990). In addition to pyroclastic material, alteration of eroded mafic volcanic material may have been important in explaining the origin of the clay minerals in mineral Association VI, as suggested by their high $\mathrm{Fe}$ and $\mathrm{Mg}$ contents (Table 3 ). The high $(\mathrm{Fe}+\mathrm{Mn}) /(\mathrm{Al}+\mathrm{Ti})$ ratios of the Lower Cretaceous sediments also suggest that ridge-crest hydrothermal activity was greater during the early stages of basin formation (Plank and Ludden, this volume). The decrease in the $(\mathrm{Fe}+\mathrm{Mn}) /(\mathrm{Al}+\mathrm{Ti})$ ratio correlates with an increase in abundance of detrital kaolinite going up the section (Fig. 7).

Illitization of smectite has been documented in the burial diagenesis of Tertiary sediments from the U.S. Gulf Coast region (Burst, 1969; Perry and Hower, 1970; Hower et al., 1976; and others). The general reaction proposed for the illitization of smectite is

$$
\text { smectite }+\mathrm{K} \text {-feldspar }=\text { illite }+ \text { chlorite }+ \text { quartz } .
$$

The possibility that the greater amount of illite in the I/S of mineral Association VII formed by illitization of smectite is supported by the following:

1. The percentage of illite layers in the I/S increases with depth from $<10 \%$ to $40 \%$, compared with the highly variable and greater amount of illite layers in detrital I/S found elsewhere at Site 765 (Fig. 7).
2. Mineral Association VII is the basal sedimentary unit at Site 765 and has experienced the highest temperatures for the greatest amount of time. Mineral Association VII also contains abundant quartz-replaced radiolarians, which is consistent with previous research that suggests that initial illitization occurs after the silica transformation of opal-CT to quartz (Compton, in press).

3. Clay-rich layers in mineral Association VII have been interpreted as metabentonites that contain I/S with $40 \%$ illite layers (Sample 123-765C-60R-5, $120 \mathrm{~cm}$ ). Although the present-day temperature of these sediments (approximately $32^{\circ} \mathrm{C}$ ) is significantly less than the temperatures of initial illitization observed in the Tertiary Gulf Coast and the Miocene Monterey Formation $\left(70^{\circ}-80^{\circ} \mathrm{C}\right)$, the greater age of mineral Association VII (140-150 Ma) may have provided enough time for illitization to occur at lower temperatures (Eberl and Hower, 1976).

Both the decrease in pore-water $\mathrm{Mg}$ and $\mathrm{K}$ and the increase in pore-water Ca with increasing sediment depth at Site 765 (Ludden, Gradstein, et al., 1990) have been commonly observed at deep-sea sites and are explained by alteration of the underlying basalt and volcanic material in the sediment (e.g., Gieskes, 1981, 1983). Explaining the unusually low pore-water chloride concentrations observed in the Lower Cretaceous sediments (mineral Association VI) at Site 765 remains problematic (Ludden, Gradstein, et al., 1990). One possible explanation is dewatering of the highly smectitic clay minerals during increasing burial diagenesis.

\section{Sepiolite-Palygorskite-Saponite or Illite/Smectite/Chlorite(?)}

The clay minerals sepiolite, palygorskite, and a clay mineral tentatively identified as either saponite or mixed-layered I/S/C 
occur in several different associations at Site 765 (Fig. 8). Sepiolite and palygorskite are $\mathrm{Mg}$-rich silicates that are most commonly found in marine sediments that are older than Eocene, with palygorskite typically far more abundant than sepiolite (Kastner, 1981). Sepiolite and palygorskite have been found to be associated with biogenic (opal-A) silica, opal-CT silica, zeolite minerals, smectite, and, in places, dolomite (von Rad and Rödsch, 1972; von Rad et al., 1977; Couture, 1977a). At Site 765, sepiolite, palygorskite, and saponite-I/S/C are associated with redeposited biogenic aragonite and diagenetic dolomite in Miocene mineral Associations II and III (Compton, this volume). In mineral Association V (Late Cretaceous), palygorskite is associated with opalA radiolarian tests and clinoptilolite. The bulk-sediment $\mathrm{FeO} /$ $\mathrm{TiO}_{2}$ ratio decreases and the $\mathrm{MgO} / \mathrm{TiO}_{2}$ ratio (Plank and Ludden, this volume) increases in the sepiolite- and palygorskite-rich mineral Associations II and III (Fig. 8), reflecting the relatively high $\mathrm{Mg}$ and low $\mathrm{Fe}$ contents of these $\mathrm{Mg}$-rich minerals.

Sepiolite and palygorskite can be either detrital or authigenic (see review by Kastner, 1981). Sepiolite has been precipitated at low temperatures and is favored by alkaline waters having high concentrations of $\mathrm{Mg}$ and $\mathrm{Si}$ (Siffert, 1962; Preisinger, 1963; Wollast et al., 1968; Couture, 1977b). It is difficult to determine if the sepiolite, palygorskite, and saponite-I/S/C are authigenic (formed in-situ), redeposited, or detrital at Site 765 . We are unaware of any detrital sources of these clay minerals from the Australian continent. Palygorskite occurs in Upper Cretaceous sediments along the entire northwestern Australia continental margin at Sites 259, 260, 261 (Cooks et al., 1974), 765, and 766 (Compton et al., this volume). The regional extent of Miocene palygorskite and sepiolite is unknown because Miocene sediments were not recovered at the other sites.

SEM photomicrographs show delicate fibers of palygorskite growing on the surface and draping diagenetic dolomite rhombs, which suggests that the palygorskite formed in situ (Pl. 3, Figs. 1 and 2). The exponential decrease in pore-water $\mathrm{Mg}$ also suggests precipitation of Mg-rich clays at depth in the sediment (Fig. 8). Pore waters at Site 765 are not particularly alkaline $(\mathrm{pH}$ ranges from 7.1 to 8.0 ), and pore-water silica is fairly low in those sediments where sepiolite and palygorskite occur, except in mineral Association V, where silica concentrations reach $600 \mu \mathrm{M}$ (Fig. 9). The low silica concentrations in mineral Associations II and III may result from the removal of pore-water silica during the formation of sepiolite and palygorskite. Radiolarian tests were not observed in mineral Associations II and III, but minor amounts of siliceous sponge rexes and spicules were observed, suggesting that some of the silica was supplied by the solution of biogenic silica. Additional Si was supplied by diffusion from the uppermost sediment, which contains abundant opal-A radiolarians (Fig. 9). $\mathrm{Mg}$ has been supplied by diffusion from the overlying seawater and also is being removed by the precipitation of dolomite (Compton, this volume). The amount of $\mathrm{Mg}$ that can be supplied will depend on the sedimentation rate or residence time of the sediment near the sediment/seawater interface, where the diffusive flux is greatest (Gieskes, 1975; Compton and Siever, 1986). Sepiolite and palygorskite commonly occur in sediments associated with hiatuses or periods of slow deposition, such as the Late Cretaceous (Couture, 1977b; Kastner, 1981). Some of the palygorskite at Site 765 occurs in slowly deposited middle to Upper

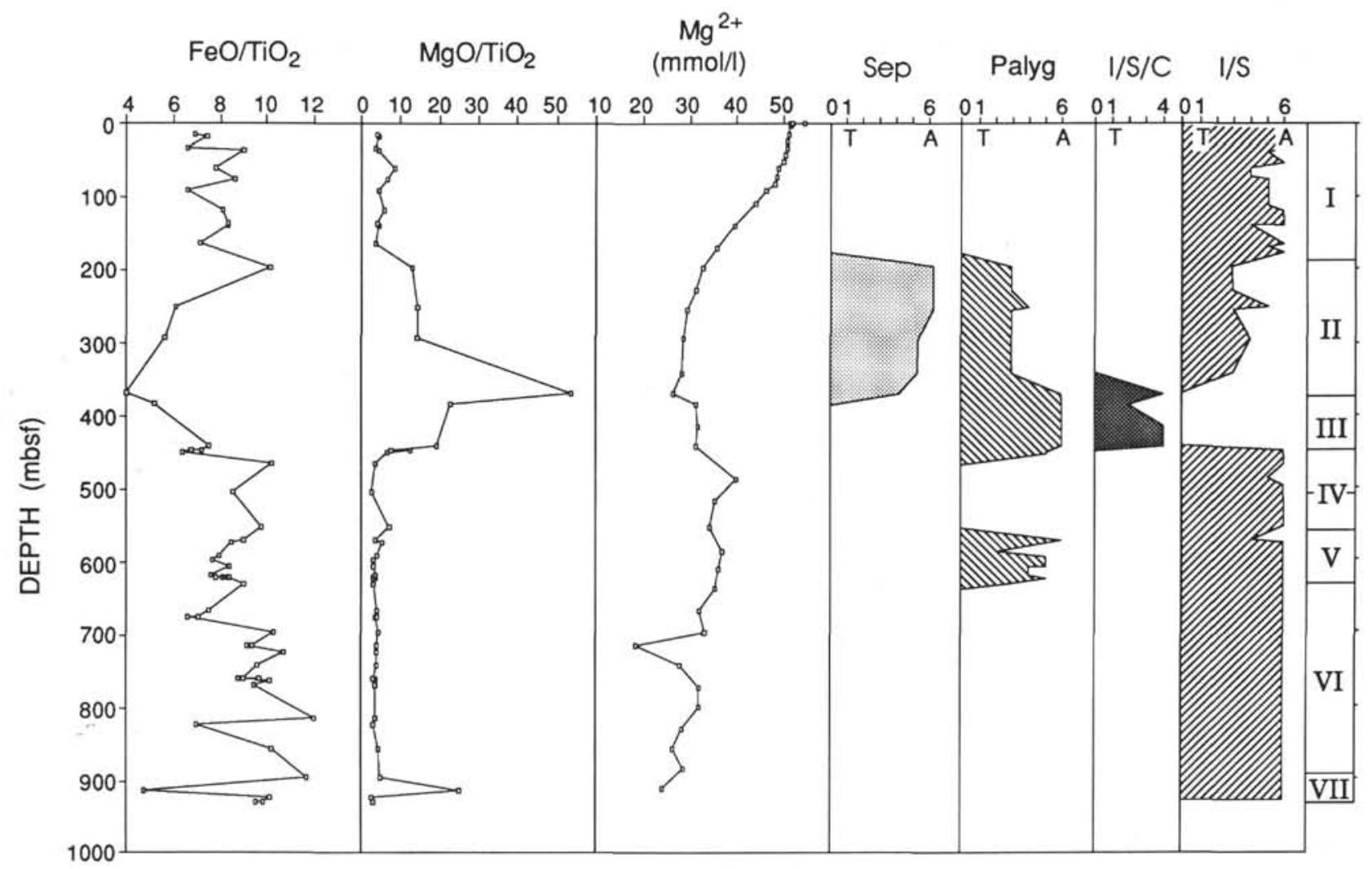

Figure 8. $\mathrm{FeO} / \mathrm{TiO}_{2}$ and $\mathrm{MgO} / \mathrm{TiO}_{2}$ ratios of the bulk sediment, pore-water $\mathrm{Mg}^{2+}$, and the relative abundances $(0=$ nondetectable, $1=$ trace (T, $<1 \mathrm{wt} \%)$ to $6=$ abundant $(\mathrm{A},>20 \mathrm{wt} \%)$ of sepiolite, palygorskite, saponite, or mixed-layered I/S/C and mixed-layered I/S with sediment depth at Site 765. Mineral Associations I through VII are shown on the far right. 


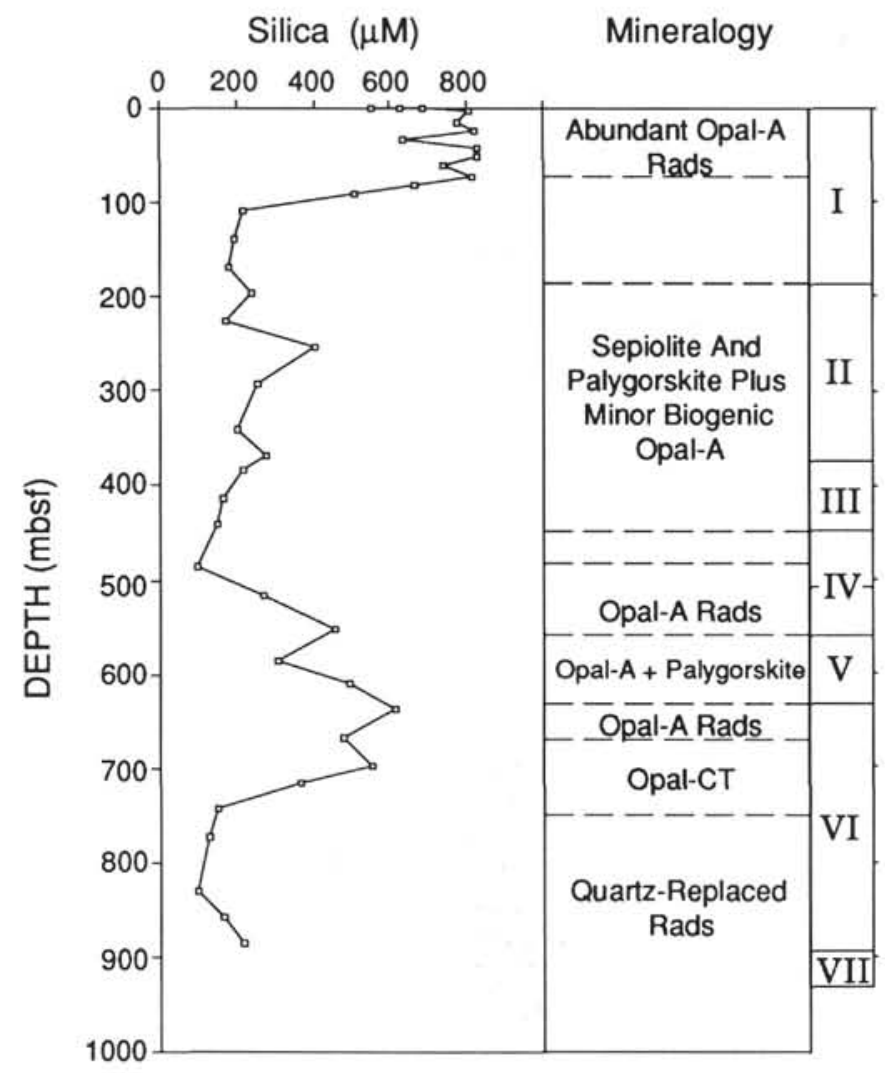

Figure 9. Pore-water silica concentration $(\mu \mathrm{M})$ and the distribution of opal-A, opal-CT, diagenetic quartz, sepiolite, and palygorskite as a function of sediment depth at Site 765. Mineral Associations I through VII are shown on the far right.

Cretaceous sediments (mineral Association V), but most of the palygorskite and all of the sepiolite occur in more rapidly deposited middle to upper Miocene turbidites.

The rapid decrease in pore-water $\mathrm{Mg}$ and $\mathrm{Si}$, the disappearance of sepiolite, and the corresponding increase in palygorskite at Site 765 all suggest that sepiolite may alter to palygorskite. The source of $\mathrm{Al}$ to form palygorskite may have been from the dissolution of associated aluminosilicate minerals and may explain why only trace-to-nondetectable amounts of feldspar minerals were observed in mineral Associations II and III. Palygorskite may also form from the alteration of volcanic ash or smectite with the addition of $\mathrm{Mg}$ and silica (Peterson et al., 1970; von Rad and Rösch, 1972; Couture, 1977b).

No previous reports of saponite- $\mathrm{I} / \mathrm{S} / \mathrm{C}$ in marine sediments are known. What was originally interpreted as the 001 chlorite peak was found to shift to higher d-spacing after glycolation to between 14.8 and $15.3 \AA$ (Fig. 5). Based on a shift in the 001 reflection and overall glycolated XRD patterns, this clay mineral was interpreted as $\mathrm{I} / \mathrm{S} / \mathrm{C}$, with a composition estimated to range from 0.33 / $0.33 / 0.33$ to $0.4 / 0.4 / 0.2$ by comparison with calculated $\mathrm{I} / \mathrm{S} / \mathrm{C}$ diffraction patterns (Reynolds, 1980). The high $\mathrm{MgO} / \mathrm{TiO}_{2}$ ratio compared with the $\mathrm{FeO} / \mathrm{TiO}_{2}$ ratio of these sediments (Fig. 8) suggests that it is a Mg-rich, as opposed to an Fe-rich, clay mineral. It is also possible that this clay mineral, interpreted as $\mathrm{I} / \mathrm{S} / \mathrm{C}$, may be saponite, a Mg-rich trioctahedral smectite. Saponite has been known to resist complete expansion to $17 \AA$ by glycolation (Robert Reynolds, pers. comm., 1991). After glycolating these samples for 1 week at $60^{\circ} \mathrm{C}$, the peaks expanded slightly to 14.9 and $15.8 \AA$, respectively, but were still less than the $17 \AA$ expected for smectitic clay minerals. It is possible that glycolation for periods greater than 1 week are required to expand saponite fully. A Mg-rich smectite such as saponite is consistent with the abundance of associated $\mathrm{Mg}$-rich sepiolite, palygorskite, and dolomite in these sediments and suggests that an abundant source of $\mathrm{Mg}$ was available during the formation of these minerals. Therefore, we interpret the unusual clay mineral of mineral Association $\mathrm{III}$ as either $\mathrm{I} / \mathrm{S} / \mathrm{C}$ or saponite and present its XRD pattern for any future reinterpretation (Fig. 5).

\section{SILICA MINERAL DIAGENESIS}

The silica phase transformations opal-A to opal-CT and opalCT to quartz occur with increasing burial depth at Site 765 (Fig. 9). The uppermost sediment ( 0 to $90 \mathrm{mbsf}$ ) contains abundant, well-preserved opal-A radiolarian tests (Ludden, Gradstein, et al., 1990). Solution of these radiolarian tests results in pore-water silica concentrations in excess of $800 \mu \mathrm{M}$ (Fig. 9). Pore-water silica decreases rapidly to $200 \mu \mathrm{M}$ by $110 \mathrm{mbsf}$. Radiolarian tests were not observed from 90 to $530 \mathrm{mbsf}$, but minor amounts of opal- $\mathrm{A}$, in the form of robust sponge rexes and spicules, were observed in places in the Neogene sediments at Site 765 and, in particular, at $254 \mathrm{mbsf}$, where a local pore-water silica maximum of $400 \mu \mathrm{M}$ occurs (Fig. 9). The paucity of biogenic silica in sediments from 90 to 530 mbsf may be a result of low surface productivity, poor preservation, or solution of the biogenic silica after burial and its precipitation as the authigenic $\mathrm{Mg}$-silicates sepiolite and palygorskite.

Pore-water silica decreases to a minimum of $100 \mu \mathrm{M}$ at the base of the Neogene sediments and then increases to a secondary maximum of about 400 to $600 \mu \mathrm{M}$ at 553 and $638 \mathrm{mbsf}$, respectively, before decreasing rapidly to 100 to $200 \mu \mathrm{M}$ in the basal sediments (Fig. 9). The local pore-water silica maxima at 553 and $638 \mathrm{mbsf}$ correspond to sediment containing significantly greater amounts of radiolarian tests (Ludden, Gradstein, et al., 1990). These radiolarian tests are thought to be composed of opal-A, because no opal-CT was detected in the XRD patterns and because the silica concentrations are too high for equilibrium with quartz. Abundant opal-CT lepispheres, as well as diagenetic quartz, occur in sediments from 670 to $744 \mathrm{mbsf}$. The transition from sediments that contain opal-CT to those containing abundant quartz-replaced radiolarian tests corresponds to a rapid decrease in porewater silica from 500 to 100 or $200 \mu \mathrm{M}$.

The temperature of the opal-CT-to-quartz transformation is estimated as $30^{\circ} \mathrm{C}$, based on the present-day geothermal gradient of about $32^{\circ} \mathrm{C} / \mathrm{km}$ measured at Site 765 (Ludden, Gradstein, et al., 1990). A temperature of $30^{\circ} \mathrm{C}$ for the opal-CT-to-quartz transformation is relatively low compared with other siliceous deposits. For example, the opal-CT-to-quartz transformation in the siliceous rocks of the Miocene Monterey Formation is on the order of $70^{\circ}$ to $80^{\circ} \mathrm{C}$ (Murata et al., 1977; Pisciotto, 1978; among others). The lower transformation temperature at Site 765 can be explained by the greater age of these sediments (ca. $120 \mathrm{Ma}$ ), which appears to have been sufficient time for the opal-CT-toquartz transformation at $30^{\circ} \mathrm{C}$, based on the experimental results of Ernst and Calvert (1969). The clay mineral content of the sediments also may have influenced the rate of these silica transformations (Kastner et al., 1977; Isaacs, 1982). The removal of pore-water $\mathrm{Mg}$ and silica during the formation of sepiolite and palygorskite, as well as the removal of pore-water $\mathrm{Mg}$ during the formation of dolomite and $\mathrm{Mg}$-rich smectite, may have inhibited the formation of opal-CT, because the availability of Mg ions has been shown in laboratory experiments to be important in the nucleation of opal-CT (Kastner et al., 1977).

\section{SUMMARY}

1. Seven distinct clay mineral associations may be defined for sediments recovered at Site 765 in the Argo Basin. These clay 
mineral associations reflect changes in burial diagenesis, lithology, and tectonic evolution of the Argo Basin. Initial basin formation was associated with volcanism and deposition of immature volcaniclastic and terrigenous sediments. Volcaniclastic sediments were altered to highly smectitic mixed-layered I/S, and volcanic ash layers were altered to bentonites and metabentonites. The illitization of smectite corresponds to the opal-CT-to-quartz transformation. The increase in abundance of kaolinite and decrease in $(\mathrm{Fe}+\mathrm{Mn}) /(\mathrm{Al}+\mathrm{Ti})$ ratio from Lower to Upper Cretaceous sediments reflect the decreasing importance of active rifting processes that included hydrothermal alteration of mid-oceanridge basalts and the deposition of large amounts of locally derived volcaniclastics.

2. Upper Cretaceous sediments from Site 765 were deposited slowly and contain palygorskite and detrital I/S that has a highly variable and large percentage of illite layers. Paleogene sediments also were deposited slowly, but contain no palygorskite. Highly smectitic I/S, zeolite minerals, and several distinct bentonite layers indicate that volcanism increased during the Paleocene and Eocene. Clay minerals from Oligocene and lower Miocene sediments are dominated by detrital I/S that contains $12 \%$ to $40 \%$ illite layers.

3. The rapidly deposited middle Miocene calcareous turbidites contain an unusual mineral association of palygorskite, dolomite, and a clay mineral tentatively identified as saponite or mixed-layered I/S/C. Upper Miocene turbidites contain sepiolite and dolomite. These Mg-rich minerals are thought to be diagenetic because of the intimate association of delicate fibers of palygorskite with euhedral dolomite crystals. $\mathrm{Mg}$ required for the formation of these $\mathrm{Mg}$-rich minerals was supplied by diffusion from the overlying seawater, and the required $\mathrm{Si}$ to form the clay minerals was supplied by the dissolution of biogenic silica.

4. Clay minerals in the Pliocene and Pleistocene calcareous turbidites contain abundant detrital I/S, kaolinite, and mica that was derived from the Australian continent, but do not contain any of the Mg-rich diagenetic clay minerals found in the underlying Miocene sediments.

\section{ACKNOWLEDGMENTS}

We gratefully acknowledge financial support from USSAC. We thank the members of the Leg 123 scientific party, technicians, and crew. T. Greco assisted with the SEM work. Robert C. Reynolds and an anonymous reviewer provided useful comments and suggestions.

\section{REFERENCES}

Biscaye, P. E., 1965. Mineralogy and sedimentation of recent deep-sea clays in the Atlantic Ocean and adjacent seas and oceans. Geol. Soc. Am. Bull., 76:803-832.

Bramlette, M. N., 1946. The Monterey Formation of California and the origin of its siliceous rocks. Geol. Surv. Prof. Pap. U.S., No. 212.

Burst, J. F., Jr., 1969. Diagenesis of Gulf Coast clayey sediments and its possible relation to petroleum migration. AAPG Bull., 53:73-93.

Compton, J. S., in press. Clay mineral diagenesis in the Monterey Formation, Santa Maria basin area, California. Clays Clay Miner.

Compton, J. S., and Siever, R., 1986. Diffusion and mass balance of $\mathrm{Mg}$ during early dolomite formation, Monterey Formation. Geochim. Cosmochim. Acta, 50:125-135.

Cook, H. E., Zemmels, I., and Matti, J. C., 1974. X-ray mineralogy data, eastern Indian Ocean-Leg 27, Deep Sea Drilling Project. In Veevers, J. J., Heirtzler, J. R., et al., Init. Repts. DSDP, 27: Washington (U.S. Govt. Printing Office), 535-548.

Couture, R. A., 1977a. Composition and origin of palygorskite-rich and montmorillonite-rich zeolite-containing sediments from the Pacific Ocean. Chem. Geol., 19:113-130.

, 1977b. Synthesis of some clay minerals at $25^{\circ} \mathrm{C}$. Palygorskite and sepiolite in the ocean [Ph.D. dissert.]. Scripps Inst. of Oceanography, La Jolla, CA.
Eberl, D., and Hower, J., 1976. Kinetics of illite formation. Geol. Soc. Am. Bull., 87:1326-1330.

Ernst, W. G., and Calvert, S. E., 1969. An experimental study of the recrystallization of porcelanite and its bearing on the origin of some bedded cherts. Am. J. Sci., 267A:114-133.

Gieskes, J. M., 1975. Chemistry of interstitial waters of marine sediments. Annu. Rev, Earth Planet. Sci., 3:433-453.

1981. Deep-sea drilling interstitial water studies: implications for chemical alteration of the oceanic crust, layers I and II. In Warme, J. E., Douglas, R. G., and Winterer, E. L. (Eds.), The Deep Sea Drilling Project: A Decade of Progress. Spec. Publ,-Soc. Econ. Paleontol. Mineral., 32:149-167.

1983. The chemistry of interstitial waters of deep-sea sediments: interpretation of Deep-Sea Drilling data. In Riley, J. P., and Chester, R. (Eds.), Chemical Oeanography (Vol. 8): London (Academic Press), 222-269.

Grim, R. E., and Güven, N., 1978. Bentonites: Geology, Mineralogy, Properties, and Uses: Amsterdam (Elsevier).

Hower, J., Eslinger, E. V., Hower, M. E., and Perry, E. A., 1976. Mechanism of burial metamorphism of argillaceous sediment: mineralogical and chemical evidence. Geol. Soc. Am. Bull., 87:725-737.

Isaacs, C. M., 1982. Influence of rock composition on kinetics of silica phase changes in the Monterey Formation, Santa Barbara area, California. Geology, 10:304-308.

Kastner, M., 1981. Authigenic silicates in deep sea sediments: formation and diagenesis. In Emiliani, C. (Ed.), The Sea (Vol. 7): New York (Wiley), 915-980.

Kastner, M., Keene, J. B., and Gieskes, J. M., 1977. Diagenesis of siliceous oozes. I. Chemical controls on the rate of opal-A to opal-CT transformation-an experimental study. Geochim. Cosmochim. Acta, 41:1041-1059.

Keller, W. D., Reynolds, R. C., and Inoue, A., 1986. Morphology of clay minerals in the smectite-to-illite conversion series by scanning electron microscopy. Clays Clay Miner., 34:187-197.

Kennett, J. P., 1982. Marine Geology: Englewood Cliffs, NJ (Prentice Hall).

Ludden, J. N., Gradstein, F. M., et al., 1990. Proc. ODP, Init. Repts., 123: College Station, TX (Ocean Drilling Program).

Moore, D. M., and Reynolds, R. C., Jr., 1989. X-ray Diffraction and the Identification and Analysis of Clay Minerals. Oxford (Oxford Univ. Press).

Murata, K. J., Friedman, I., and Gleason, J. D., 1977. Oxygen isotope relations between diagenetic silica minerals in Monterey Shale, Temblor Range, California. Am. J. Sci., 277:259-272.

Newman, A.C.D. and Brown, G., 1987. The chemical constitution of clays. In Newman, A.C.D. (Ed.), Chemistry of Clays and Clay Minerals: Mineral. Soc. Monogr., 6:1-128.

Ninkovich, D., 1979. Distribution, age and chemical composition of tephra layers in deep-sea sediments off Western Indonesia. J. Volcanol. Geotherm. Res., 5:67-86.

Perry, E. A., Jr., and Hower, J., 1970. Burial diagenesis of the Gulf Coast pelitic sediments. Clays Clay Miner., 18:195-177.

Peterson, M.N.A., Edgar, N. T., von der Borch, C. C., and Rex, R. W., 1970. Cruise leg summary and discussion. In Peterson, M.N.A., Edgar, N. T., et al., Init. Repts. DSDP, 2: Washington (U.S. Govt. Printing Office), 413-427.

Pisciotto, K. A., 1978. Basinal sedimental facies and diagenetic aspects of the Monterey shale, California [Unpubl. Ph.D. dissert.]. Univ. of California, Santa Cruz.

Preisinger, A., 1963. Sepiolite and related compounds: its stability and application. Clays Clay Miner., 10:365-371.

Reynolds, R. C., Jr., 1980. Interstratified clay minerals. In Brindley, G. W., and Brown, G. (Eds.), Crystal Structures of Clay Minerals and their X-Ray Identification. Mineral. Soc. London Monogr., 5:249303.

Reynolds, R. C., Jr., and Hower, J., 1970. The nature of interlayering in mixed-layer illite-montmorillonite. Clays Clay Miner., 18:25-36.

Siffert, B., 1962. Synthèse d'une sépiolite à température ordinaire. C. $R$. Seances Acad. Sci., 254:1460-1462.

von Rad, U., Riech, V., and Rösch, H., 1977. Silica diagenesis in continental margin sediments off northwest Africa. In Lancelot, Y., and Seibold, E., et al., Init. Repts. DSDP, 41: Washington (U.S. Govt. Printing Office), 879-905. 
von Rad, U., and Rösch, H., 1972. Mineralogy and origin of clay minerals, silica and authigenic silicates in Leg 14 sediments. In Hayes, D. E., Pimm, A. C., et al., Init. Repts. DSDP, 14: Washington (U.S. Govt. Printing Office), 727-746.

von Rad, U., and Thurow, J., in press. Bentonitic clays as indicators of early Neocomian post-breakup volcanism off NW Australia (ODP Leg 122). In von Rad, U., Haq, B. U., et al., Proc. ODP, Sci. Results, 122: College Station, TX (Ocean Drilling Program).
Wollast, R., Mackenzic, F. T., and Bricker, O. P., 1968. Experimental precipitation and genesis of sepiolite earth surface conditions. Am. Mineral., 53:1645-1662.

Date of initial receipt: 9 July 1990

Date of acceptance: 19 March 1991

Ms 123B-164 


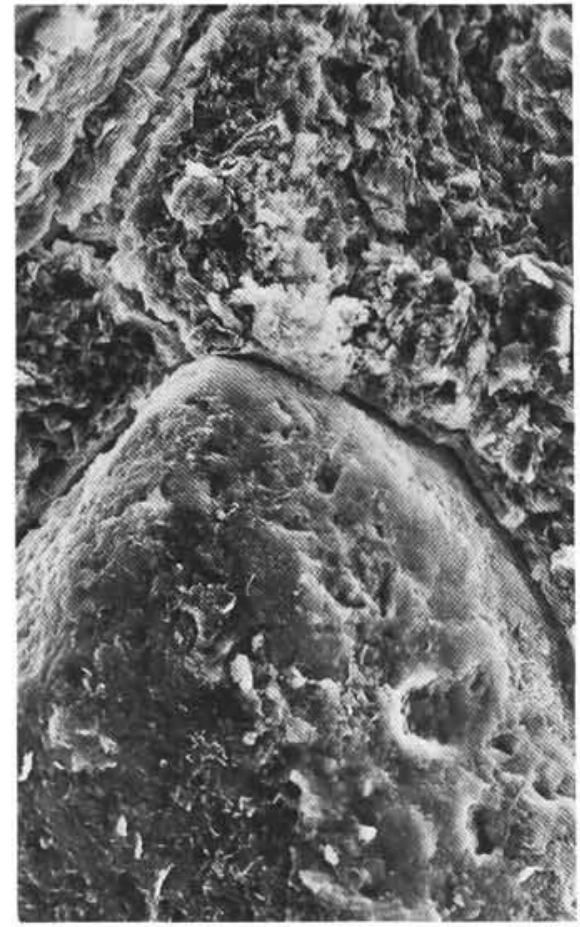

1

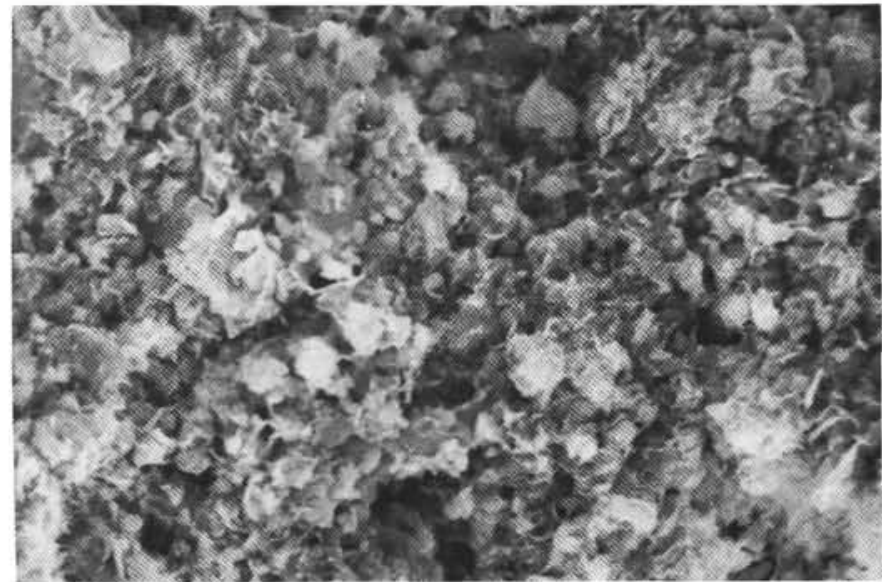

3 $\overline{10 \mu \mathrm{m}}$

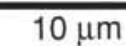

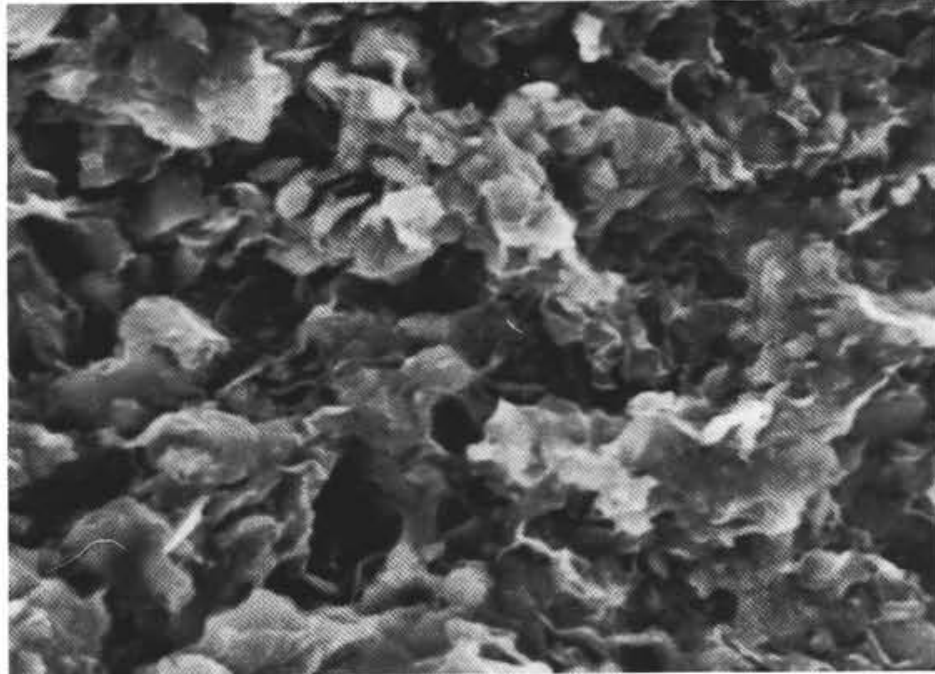

2

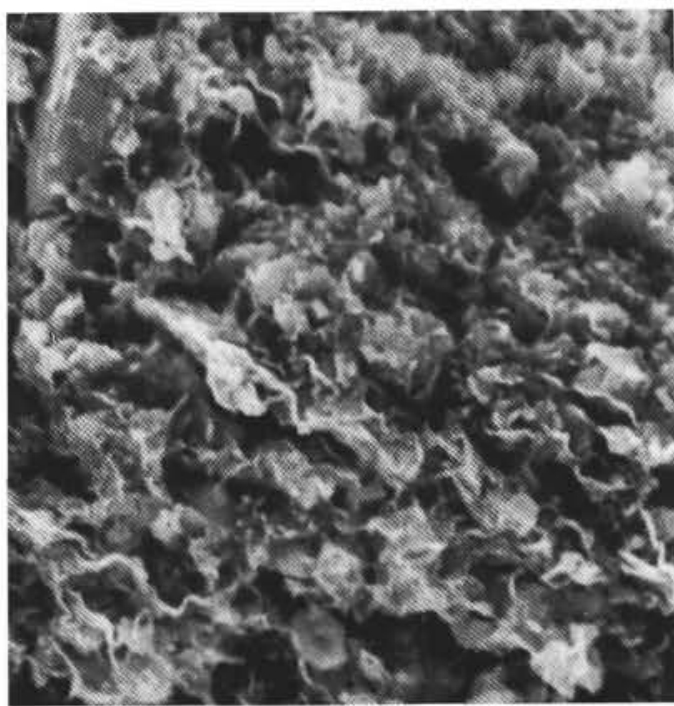

4

$10 \mu \mathrm{m}$

Plate 1. SEM photomicrographs of sediments from Site 765. 1. Overview of contact between detrital grain (volcaniclastic?) and mixed-layered illite/smectite (I/S) matrix in Sample 123-765C-61R-5, 81-91 cm, from mineral Association VII. 2. Close-up of scallop-shaped flakes of I/S having approximately $40 \%$ illite layers, Sample $123-765 \mathrm{C}-61 \mathrm{R}-5,81-91 \mathrm{~cm}$. 3. Overview of highly smectitic I/S typical of mineral Association VI, Sample 123-765C-51R-3, 140-150 cm. 4. Close-up of I/S containing less than $10 \%$ illite layers, Sample $123-765 \mathrm{C}-51 \mathrm{R}-3,140-150 \mathrm{~cm}$. Scale bars $=10 \mu \mathrm{m}$. 

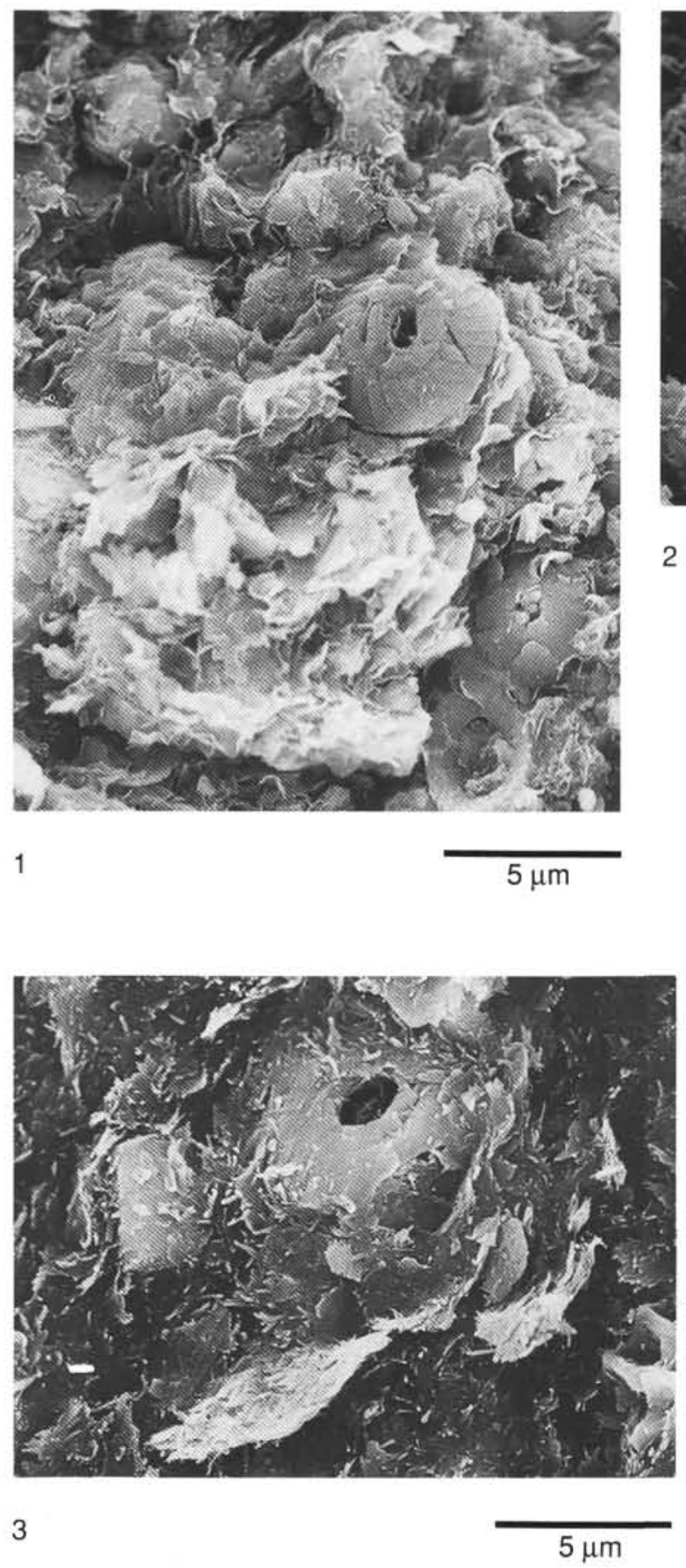

2

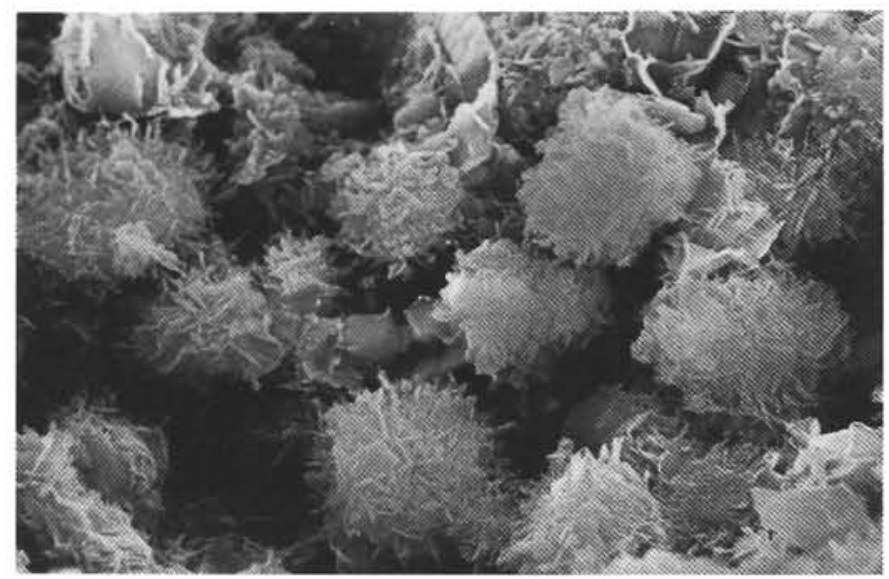

$5 \mu \mathrm{m}$

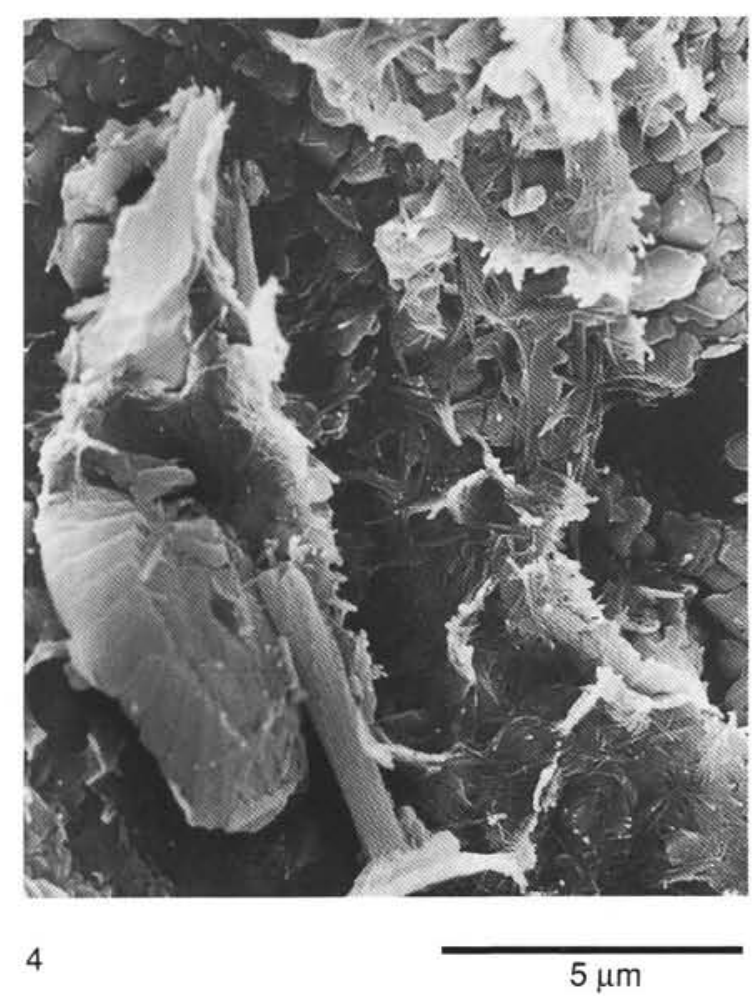

Plate 2. SEM photomicrographs of sediments from Site 765. 1. Mixed-layered I/S and coccoliths, Sample $123-765 \mathrm{C}-34 \mathrm{R}-3,115 \mathrm{~cm}$, from mineral Association VI. 2. Close-up of I/S and opal-CT lepispheres, Sample 123-765C-34R-3, $115 \mathrm{~cm}$. 3. Clay minerals (palygorskite and saponite or mixed-layered I/S/C?), coccolith, and dolomite rhomb left of coccolith, Sample 123-765C-5R-4, 39-41 cm, from mineral Association III. 4. Fibrous palygorskite, coccoliths, and recrystallized carbonate, Sample $123-765 \mathrm{C}-5 \mathrm{R}-4,39-41 \mathrm{~cm}$. Scale bars $=5 \mu \mathrm{m}$. 


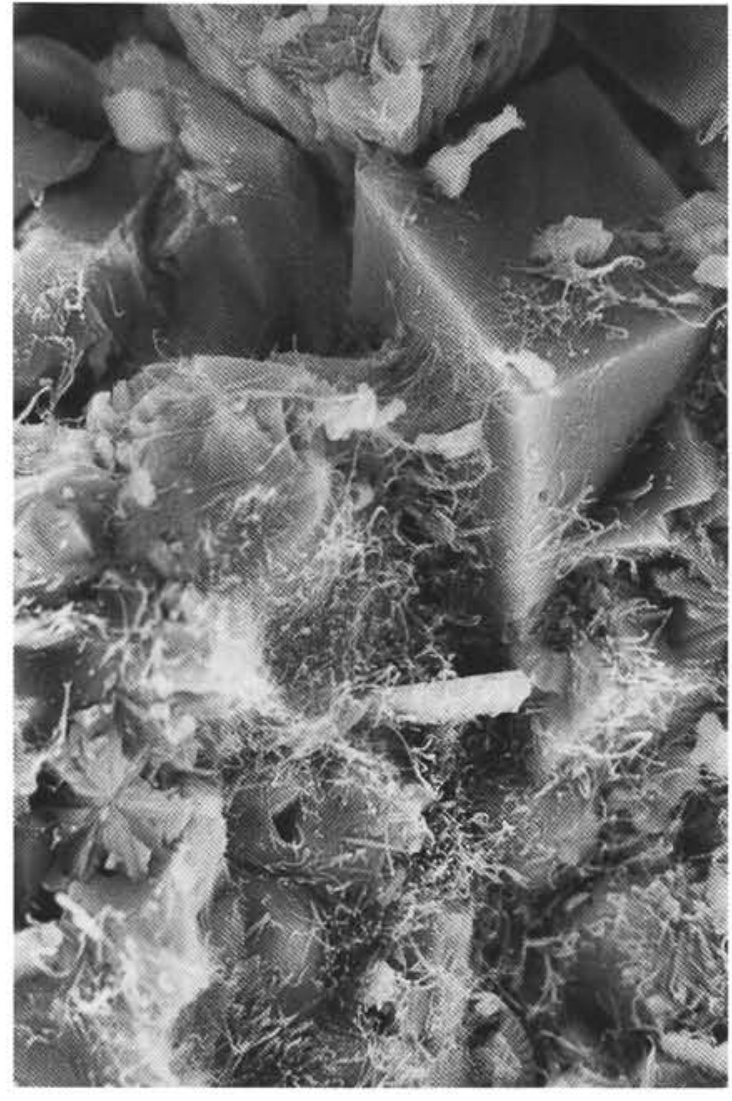

1

$5 \mu \mathrm{m}$

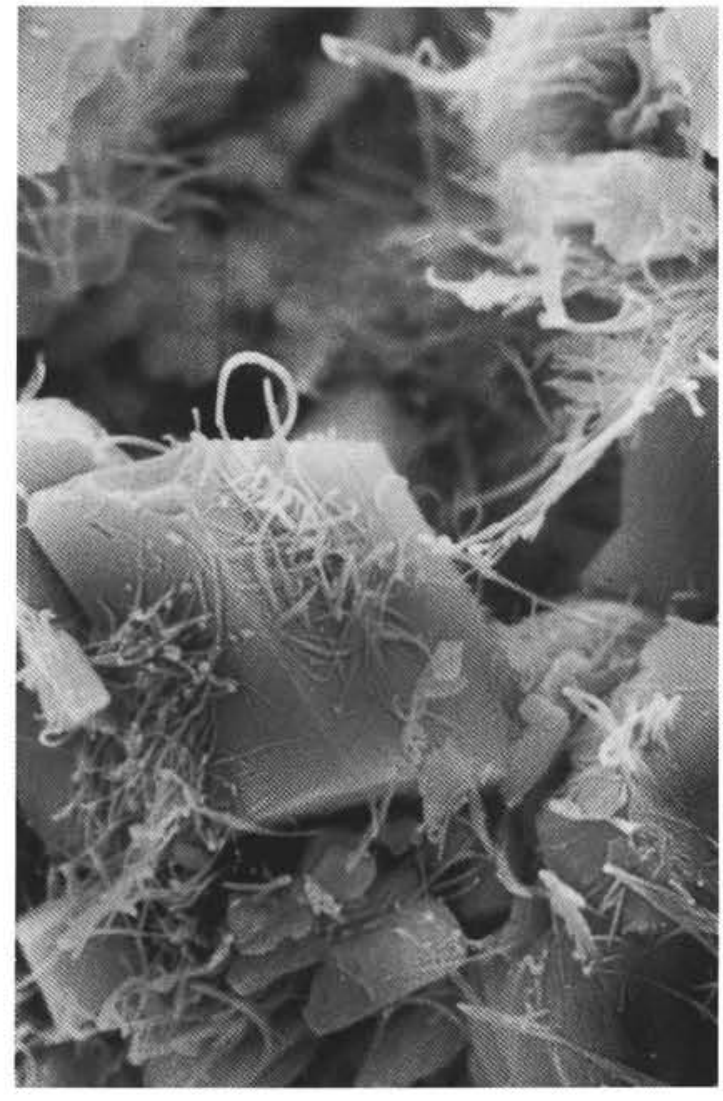

2

Plate 3. SEM photomicrographs of fibrous palygorskite on the surfaces of rhombic dolomite crystals, Sample $123-765 \mathrm{C}-4 \mathrm{R}-3,140-150 \mathrm{~cm}$, from mineral Association III. 1. Scale bar $=5 \mu \mathrm{m}$. 2. Scale bar $=1 \mu \mathrm{m}$. 